\title{
New Triterpene Glycosides from the Far Eastern Starfish Solaster pacificus and Their Biological Activity
}

\author{
Timofey V. Malyarenko ${ }^{1,2, *}$, Alla A. Kicha ${ }^{1}$, Anatoly I. Kalinovsky ${ }^{1}$, Pavel S. Dmitrenok ${ }^{1}{ }^{(\mathbb{D}}$, \\ Olesya S. Malyarenko ${ }^{1}{ }^{(0)}$, Alexandra S. Kuzmich ${ }^{1}$, Valentin A. Stonik ${ }^{1,2}$ and Natalia V. Ivanchina ${ }^{1}$ \\ 1 G.B. Elyakov Pacific Institute of Bioorganic Chemistry, Far Eastern Branch of the Russian Academy of \\ Sciences, Pr. 100-let Vladivostoku 159, 690022 Vladivostok, Russia; kicha@piboc.dvo.ru (A.A.K.); \\ kaaniw@piboc.dvo.ru (A.I.K.); paveldmt@piboc.dvo.ru (P.S.D.); malyarenko.os@gmail.com (O.S.M.); \\ assavina@mail.ru (A.S.K.); stonik@piboc.dvo.ru (V.A.S.); ivanchina@piboc.dvo.ru (N.V.I.) \\ 2 Department of Bioorganic Chemistry and Biotechnology, School of Natural Sciences, \\ Far Eastern Federal University, Sukhanova str. 8, 690000 Vladivostok, Russia \\ * Correspondence: malyarenko-tv@mail.ru; Tel.: +7-423-2312-360; Fax: +7-423-2314-050
}

check for updates

Citation: Malyarenko, T.V.; Kicha, A.A.; Kalinovsky, A.I.; Dmitrenok, P.S.; Malyarenko, O.S.; Kuzmich, A.S.; Stonik, V.A.; Ivanchina, N.V. New Triterpene Glycosides from the Far Eastern Starfish Solaster pacificus and Their Biological Activity. Biomolecules 2021, 11, 427. https://doi.org/ 10.3390/biom11030427

Academic Editor: Elena Leychenko

Received: 16 February 2021

Accepted: 11 March 2021

Published: 14 March 2021

Publisher's Note: MDPI stays neutral with regard to jurisdictional claims in published maps and institutional affiliations.

Copyright: (c) 2021 by the authors. Licensee MDPI, Basel, Switzerland. This article is an open access article distributed under the terms and conditions of the Creative Commons Attribution (CC BY) license (https:/ / creativecommons.org/licenses/by/ $4.0 /)$.

\begin{abstract}
Three new triterpene glycosides, pacificusosides A-C (1-3), and three previously known triterpene glycosides, cucumariosides $C_{1}(4), C_{2}(5)$, and $A_{10}(6)$, were isolated from the alcoholic extract of the Far Eastern starfish Solaster pacificus. The structures of 1-3 were elucidated by extensive NMR and ESIMS techniques and chemical transformations. Compound $\mathbf{1}$ has a novel, unique structure, containing an aldehyde group of side chains in its triterpene aglycon. This structural fragment has not previously been found in the sea cucumber triterpene glycosides or starfish steroidal glycosides. Probably, pacificusoside A (1) is a product of the metabolism of the glycoside obtained through dietary means from a sea cucumber in the starfish. Another two new triterpene glycosides $(2,3)$ have closely related characteristics to sea cucumber glycosides. The cytotoxicity of compounds 1-6 was tested against human embryonic kidney HEK 293 cells, colorectal carcinoma HT-29 cells, melanoma RPMI-7951 cells, and breast cancer MDA-MB-231 cells using MTS assay. Compounds 4-6 revealed the highest cytotoxic activity against the tested cell lines, while the other investigated compounds had moderate or slight cytotoxicity. The cytotoxic effects of 2-6 were reduced by cholesterol like the similar effects of the previously investigated individual triterpene glycosides. Compounds 3, 4, and 5 almost completely suppressed the colony formation of the HT-29, RPMI-7951, and MDA-MB-231 cells at a nontoxic concentration of $0.5 \mu \mathrm{M}$.
\end{abstract}

Keywords: triterpene glycosides; starfish; Solaster pacificus; biosynthesis; food markers; cytotoxic activity; colony formation

\section{Introduction}

The phylum Echinodermata includes five classes: Holothuroidea (sea cucumbers), Asteroidea (starfish), Echinoidea (sea urchins), Crinoidea (sea lilies), and Ophiuroidea (brittle stars and basket stars). These invertebrates are a rich source of a variety of low molecular metabolites with potent biological activities: triterpene glycosides, peptides, fatty acids, polar steroids and their glycosides, carotenoids, quinones, spinochromes pigments, and also sphingolipids and their derivatives [1].

Triterpene glycosides are considered to be characteristic secondary metabolites and chemotaxonomic markers of sea cucumbers. They are amphiphilic compounds consisting of lipophilic aglycon and a hydrophilic oligosaccharide chain [2]. Usually, these glycosides contain holostane type of aglycons having lanostane-3 $\beta$-ol with $(18,20)$-lactone in the E-ring of the pentacyclic triterpene core. Some triterpene glycosides contain nonholostane aglycons [3]. As a rule, their carbohydrate chains include five or six monosaccharides such as D-xylose, D-quinovose, and D-glucose or their methylated or sulfated derivatives attached to C-3 of the aglycon. In the carbohydrate chains, the first sugar unit is always xylose, whereas 3-O-methyl-D-glucose and/or 
3-O-methyl-D-xylose are predominantly the terminal monosaccharide units. These glycosides have been reported to exhibit a wide spectrum of biological activities such as cytotoxic [4,5], antifungal [6,7], bactericidal, hemolytic, antiviral, and antiparasitic properties [8]. Some triterpene glycosides were found to induce apoptosis and inhibit the growth of cancer cells [9], and show immunomodulatory properties [10].

At the same time, polar steroids and their glycosides are characteristic secondary metabolites of starfish (sea stars). The starfish steroidal glycosides may be divided into three major classes: glycosides of polyhydroxysteroids, cyclic glycosides, and asterosaponins [11-19]. Polyhydroxysteroidal glycosides from starfish have highly oxygenated steroidal aglycon. In most cases, monosaccharides of these compounds are represented by D-xylose and L-arabinose or their methylated or sulfated derivatives, forming monoside, bioside and/or sometimes trioside carbohydrate chains. Cyclic glycosides have several unusual structural features including a trisaccharide carbohydrate chain cyclized between C-3 and C-6 of the $\Delta^{7}-3 \beta, 6 \beta-$ dihydroxysteroidal aglycon as well as the presence of a glucuronic acid unit in the carbohydrate moiety. The majority of asterosaponins contain 3-O-sulfonated $\Delta^{9(11)}-3 \beta, 6 \alpha$-dihydroxysteroidal aglycon and carbohydrate chains, comprising usually five or six monosaccharide residues attached at the C-6 position of an aglycon.

It is worth noting that triterpene glycosides are not typical secondary metabolites of starfish. Earlier only two new triterpene glycosides, rollentosides A and B were isolated from the starfish Asterias rollestoni Bell, collected in the Yellow Sea near the shore of Jiangsu Province [20]. In addition, one previously known triterpene glycoside, holothurin $\mathrm{A}_{2}$ (= echinoside $\mathrm{A}$ ), was isolated from the tropical starfish Choriaster granulatus [21]. In all other starfish triterpene glycosides were not found.

Herein, we report the results of our studies on triterpene glycosides from the ethanolic extract of the Far Eastern carnivorous starfish Solaster pacificus (order Valvatida, family Solasteridae) collected in the Sea of Okhotsk near Iturup Island. As a result, we have established the structures of three new triterpene glycosides, pacificusosides A-C (1-3), derived from the diet of this starfish. Moreover, we examined the cytotoxic activity of the individual compounds 1-6 or 1-6 in combination with cholesterol in human embryonic kidney, colorectal carcinoma, melanoma, and breast cancer cells. The effects of these compounds on the colony formation of the tested cancer cells were investigated using soft agar assay.

\section{Materials and Methods}

\subsection{General Methods}

Optical rotations were determined on a PerkinElmer 343 polarimeter (PerkinElmer, Waltham, MA, USA). UV spectra were recorded on a Shimadzu UV-1601 PC spectrophotometer (Shimadzu, Kioto, Japan). IR spectra were recorded using a Bruker Equinox 55 spectrophotometer (Bruker, Göttingen, Germany). The ${ }^{1} \mathrm{H}$ and ${ }^{13} \mathrm{C}$ NMR spectra were otained on Bruker Avance III 700 spectrometer (Bruker BioSpin, Bremen, Germany) at 700.13 and $176.04 \mathrm{MHz}$, respectively, chemical shifts were referenced to the corresponding residual solvent signals $\left(\delta_{\mathrm{H}} 3.30 / \delta_{\mathrm{C}} 49.0\right.$ for $\mathrm{CD}_{3} \mathrm{OD}$ ). The HRESIMS spectra were recorded on a Bruker Impact II Q-TOF mass spectrometer (Bruker, Bremen, Germany); the samples were dissolved in $\mathrm{MeOH}$ (c $0.001 \mathrm{mg} / \mathrm{mL}$ ). HPLC separations were carried out on an Agilent 1100 Series chromatograph (Agilent Technologies, Santa Clara, CA, USA) equipped with a differential refractometer; Diasfer-110-C18 $(10 \mu \mathrm{m}$, $250 \times 15 \mathrm{~mm}$, Biochemmack, Moscow, Russia) and Discovery $C_{18}(5 \mu \mathrm{m}, 250 \times 4 \mathrm{~mm}$, Supelco, North Harrison, PA, USA) columns were used. Low-pressure liquid column chromatography was carried out with Polychrom-1 (powdered Teflon, 0.25-0.50 mm; Biolar, Olaine, Latvia), Si

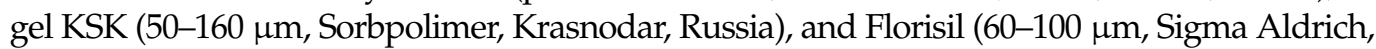
St. Louis, MO, USA). Sorbfil Si gel plates $(4.5 \times 6.0 \mathrm{~cm}, 5-17 \mu \mathrm{m}$, Sorbpolimer, Krasnodar, Russia) were used for thin-layer chromatography.

\subsection{Animal Material}

Specimens of S. pacificus Djakonov, 1938 (order Valvatida, family Solasteridae) were collected at a depth of $10 \mathrm{~m}-20 \mathrm{~m}$ by scuba diving in the Sea of Okhotsk near Iturup Island 
during the 42nd scientific cruise of the research vessel Akademik Oparin in August 2012. Species identification was carried out by Mr. B.B. Grebnev (G.B. Elyakov Pacific Institute of Bioorganic Chemistry of the FEB RAS, Vladivostok, Russia). A voucher specimen (no. 042-112) is on deposit at the marine specimen collection of the G.B. Elyakov Pacific Institute of Bioorganic Chemistry of the FEB RAS, Vladivostok, Russia.

\subsection{Extraction and Isolation}

The fresh animals $(1.7 \mathrm{~kg})$ were chopped and extracted twice with $\mathrm{EtOH}$ at $20^{\circ} \mathrm{C}$. The $\mathrm{H}_{2} \mathrm{O} / \mathrm{EtOH}$ layer was evaporated, and the residue was dissolved in $\mathrm{H}_{2} \mathrm{O}(0.5 \mathrm{~L})$. The $\mathrm{H}_{2} \mathrm{O}$-soluble materials were passed through a Polychrom- 1 column $(6.5 \times 21 \mathrm{~cm})$, eluted with distilled $\mathrm{H}_{2} \mathrm{O}$ until a negative chloride ion reaction was obtained, and eluted with $\mathrm{EtOH}$. The combined $\mathrm{EtOH}$ eluate was evaporated to give a brownish residue ( $48 \mathrm{~g})$. These materials were chromatographed over a Si gel column $(6 \times 22 \mathrm{~cm})$ using $\mathrm{CHCl}_{3} / \mathrm{EtOH}$ (stepwise gradient, 8:1 to 1:1), $\mathrm{EtOH}$, and $\mathrm{EtOH} / \mathrm{H}_{2} \mathrm{O}(4: 1,2: 1$, and 1:2) to yield fifteen fractions, 1-15, that were then analyzed by TLC on Si gel plates in the eluent system $\mathrm{BuOH} / \mathrm{EtOH} / \mathrm{H}_{2} \mathrm{O}$ (4:1:2). Fractions 4 (11.49 g) and 5 (22.79 g) were additionally chromatographed over a Florisil column $(4.5 \times 12 \mathrm{~cm})$ using $\mathrm{CHCl}_{3} / \mathrm{EtOH}$ (stepwise gradient, $5: 1$ to $1: 1)$ to yield three fractions, $41(1.459 \mathrm{~g}), 42(1.13 \mathrm{~g})$, and $51(652 \mathrm{mg})$. HPLC separation of fractions 41, 42, and 51 on a Diasfer-110-C18 column $(10 \mu \mathrm{m}, 250 \times 15 \mathrm{~mm}, 2.5 \mathrm{~mL} / \mathrm{min})$ with $\mathrm{EtOH} / \mathrm{H}_{2} \mathrm{O}$ (55:45) as an eluent system yielded pure $2\left(31 \mathrm{mg}, t_{\mathrm{R}} 25.8 \mathrm{~min}\right), 3(13.0 \mathrm{mg}$, $\left.t_{\mathrm{R}} 91.5 \mathrm{~min}\right), 4\left(89.0 \mathrm{mg}, t_{\mathrm{R}} 57.8 \mathrm{~min}\right), 5\left(59 \mathrm{mg}, t_{\mathrm{R}} 71.2 \mathrm{~min}\right)$, and subfractions $41-3(30 \mathrm{mg})$ and 42-1 (94.5 mg). HPLC separation of subfractions 41-3 and 42-1 on a Discovery C18 column $(5 \mu \mathrm{m}, 250 \times 10 \mathrm{~mm}, 2.5 \mathrm{~mL} / \mathrm{min})$ with $\mathrm{EtOH} / \mathrm{H}_{2} \mathrm{O}$ (50:50) as an eluent system yielded pure $1\left(12.5 \mathrm{mg}, t_{\mathrm{R}} 6.6 \mathrm{~min}\right)$ and $6\left(3.0 \mathrm{mg}, t_{\mathrm{R}} 16.3 \mathrm{~min}\right)$.

\subsection{Spectral Data of New Compounds}

Pacificusoside $A$ (1), $\mathrm{C}_{57} \mathrm{H}_{86} \mathrm{O}_{27}$, white amorphous powder; $[\alpha]_{\mathrm{D}}{ }^{25}-27.7$ (c $\left.0.23, \mathrm{MeOH}\right)$; $\mathrm{UV}(\mathrm{MeOH}) \lambda_{\max } 199,224 \mathrm{~nm}$; IR (KBr): $v_{\max }=3438,2927,1745,1688,1633,1422,1384,1243$, 1160, 1113, 1059, $894 \mathrm{~cm}^{-1} ;{ }^{1} \mathrm{H}$ and ${ }^{13} \mathrm{C}$ NMR data are listed in Tables 1 and 2; (+)ESIMS/MS of the ion $[\mathrm{M}+\mathrm{Na}]^{+}$at $m / z$ 1225: $1093\left[(\mathrm{M}+\mathrm{Na})-\mathrm{C}_{5} \mathrm{H}_{8} \mathrm{O}_{4}\right]^{+} ; 947\left[(\mathrm{M}+\mathrm{Na})-\mathrm{C}_{6} \mathrm{H}_{10} \mathrm{O}_{4}-\mathrm{C}_{5} \mathrm{H}_{8} \mathrm{O}_{4}\right]^{+} ; 759$ $[(\text { carbohydrate chain }+\mathrm{Na})]^{+} ; 609$ [(carbohydrate chain $\left.\left.+\mathrm{Na}\right)-\mathrm{C}_{5} \mathrm{H}_{8} \mathrm{O}_{4}-\mathrm{H}_{2} \mathrm{O}\right]^{+} ; 477$ [(carbohydrate chain $+\mathrm{Na}$ ) $\left.-2 \times \mathrm{C}_{5} \mathrm{H}_{8} \mathrm{O}_{4}-\mathrm{H}_{2} \mathrm{O}\right]^{+} ; 331$ [(carbohydrate chain $\left.\left.+\mathrm{Na}\right)-2 \times \mathrm{C}_{5} \mathrm{H}_{8} \mathrm{O}_{4}-\mathrm{H}_{2} \mathrm{O}-\mathrm{C}_{6} \mathrm{H}_{10} \mathrm{O}_{4}\right]^{+}$; $185\left[\mathrm{C}_{6} \mathrm{H}_{10} \mathrm{O}_{5}+\mathrm{Na}\right]^{+} ; 169\left[\mathrm{C}_{6} \mathrm{H}_{10} \mathrm{O}_{4}+\mathrm{Na}\right]^{+}$; (+)HRESIMS m/z $1225.5273[\mathrm{M}+\mathrm{Na}]^{+}$(calcd for $\left.\left[\mathrm{C}_{57} \mathrm{H}_{86} \mathrm{O}_{27} \mathrm{Na}\right]^{+}, 1225.5249\right) ;(-) \mathrm{ESIMS} / \mathrm{MS}$ of the ion $[\mathrm{M}-\mathrm{H}]^{-}$at $\mathrm{m} / \mathrm{z}$ 1201: 1069 [(M-H)$\left.\mathrm{C}_{5} \mathrm{H}_{8} \mathrm{O}_{4}\right]^{-} ; 923\left[(\mathrm{M}-\mathrm{H})-\mathrm{C}_{6} \mathrm{H}_{10} \mathrm{O}_{4}-\mathrm{C}_{5} \mathrm{H}_{8} \mathrm{O}_{4}\right]^{-} ; 761\left[(\mathrm{M}-\mathrm{H})-\mathrm{C}_{6} \mathrm{H}_{10} \mathrm{O}_{5}-\mathrm{C}_{6} \mathrm{H}_{10} \mathrm{O}_{4}-\mathrm{C}_{5} \mathrm{H}_{8} \mathrm{O}_{4}\right]^{-} ; 615$ $\left[(\mathrm{M}-\mathrm{H})-\mathrm{C}_{6} \mathrm{H}_{10} \mathrm{O}_{5}-2 \times \mathrm{C}_{6} \mathrm{H}_{10} \mathrm{O}_{4}-\mathrm{C}_{5} \mathrm{H}_{8} \mathrm{O}_{4}\right]^{-} ; 483\left[(\mathrm{M}-\mathrm{H})-\mathrm{C}_{6} \mathrm{H}_{10} \mathrm{O}_{5}-2 \times \mathrm{C}_{6} \mathrm{H}_{10} \mathrm{O}_{4}-2 \times \mathrm{C}_{5} \mathrm{H}_{8} \mathrm{O}_{4}\right]^{-}$; $(-)$ HRESIMS $m / z 1201.5318[\mathrm{M}-\mathrm{H}]^{-}$(calcd for $\left[\mathrm{C}_{57} \mathrm{H}_{85} \mathrm{O}_{27}\right]^{-}, 1201.5284$ ).

Pacificusoside $B$ (2), $\mathrm{C}_{53} \mathrm{H}_{82} \mathrm{O}_{24}$, white amorphous powder; $[\alpha]_{\mathrm{D}}{ }^{25}-28.0$ (c $0.1, \mathrm{MeOH}$ ); IR $(\mathrm{KBr}): v_{\max }=3427,2926,2360 ; 1772,1635,1558,1541,1384,1507,1457,1384,1180$, $1074 \mathrm{~cm}^{-1} ;{ }^{1} \mathrm{H}$ and ${ }^{13} \mathrm{C}$ NMR data are listed in Tables 1 and 2; (+)ESIMS/MS of the ion $[\mathrm{M}+\mathrm{Na}]^{+}$at $m / z$ 1125: $993\left[(\mathrm{M}+\mathrm{Na})-\mathrm{C}_{5} \mathrm{H}_{8} \mathrm{O}_{4}\right]^{+} ; 979\left[(\mathrm{M}+\mathrm{Na})-\mathrm{C}_{6} \mathrm{H}_{10} \mathrm{O}_{4}\right]^{+} ; 847[(\mathrm{M}+\mathrm{Na})-$ $\left.\mathrm{C}_{6} \mathrm{H}_{10} \mathrm{O}_{4}-\mathrm{C}_{5} \mathrm{H}_{8} \mathrm{O}_{4}\right]^{+} ; 817\left[(\mathrm{M}+\mathrm{Na})-\mathrm{C}_{6} \mathrm{H}_{10} \mathrm{O}_{4}-\mathrm{C}_{6} \mathrm{H}_{10} \mathrm{O}_{5}\right]^{+} ; 759$ [(carbohydrate chain $\left.+\mathrm{Na}\right]^{+}$; 609 [(carbohydrate chain $\left.+\mathrm{Na})-\mathrm{C}_{5} \mathrm{H}_{8} \mathrm{O}_{4}-\mathrm{H}_{2} \mathrm{O}\right]^{+} ; 477$ [(carbohydrate chain $\left.+\mathrm{Na}\right)-2 \times \mathrm{C}_{5} \mathrm{H}_{8} \mathrm{O}_{4}{ }^{-}$ $\left.\mathrm{H}_{2} \mathrm{O}\right]^{+} ; 331$ [(carbohydrate chain $\left.\left.+\mathrm{Na}\right)-2 \times \mathrm{C}_{5} \mathrm{H}_{8} \mathrm{O}_{4}-\mathrm{H}_{2} \mathrm{O}-\mathrm{C}_{6} \mathrm{H}_{10} \mathrm{O}_{4}\right]^{+} ; 185\left[\mathrm{C}_{6} \mathrm{H}_{10} \mathrm{O}_{5}+\mathrm{Na}\right]^{+}$; $169\left[\mathrm{C}_{6} \mathrm{H}_{10} \mathrm{O}_{4}+\mathrm{Na}\right]^{+} ;(+)$HRESIMS $\mathrm{m} / z 1125.5091[\mathrm{M}+\mathrm{Na}]^{+}$(calcd for $\left[\mathrm{C}_{53} \mathrm{H}_{82} \mathrm{O}_{24} \mathrm{Na}\right]^{+}$, 1125.5088); (-)ESIMS/MS of the ion [M-H] $]^{-}$at $m / z$ 1101: $969\left[(\mathrm{M}-\mathrm{H})-\mathrm{C}_{5} \mathrm{H}_{8} \mathrm{O}_{4}\right]^{-} ; 955[(\mathrm{M}-$ $\left.\mathrm{H})-\mathrm{C}_{6} \mathrm{H}_{10} \mathrm{O}_{4}\right]^{-} ; 823\left[(\mathrm{M}-\mathrm{H})-\mathrm{C}_{6} \mathrm{H}_{10} \mathrm{O}_{4}-\mathrm{C}_{5} \mathrm{H}_{8} \mathrm{O}_{4}\right]^{-} ; 793\left[(\mathrm{M}-\mathrm{H})-\mathrm{C}_{6} \mathrm{H}_{10} \mathrm{O}_{4}-\mathrm{C}_{6} \mathrm{H}_{10} \mathrm{O}_{5}\right]^{-} ; 661$ $\left[(\mathrm{M}-\mathrm{H})-\mathrm{C}_{6} \mathrm{H}_{10} \mathrm{O}_{5}-\mathrm{C}_{6} \mathrm{H}_{10} \mathrm{O}_{4}-\mathrm{C}_{5} \mathrm{H}_{8} \mathrm{O}_{4}\right]^{-} ; 515\left[(\mathrm{M}-\mathrm{H})-\mathrm{C}_{6} \mathrm{H}_{10} \mathrm{O}_{5}-2 \times \mathrm{C}_{6} \mathrm{H}_{10} \mathrm{O}_{4}-\mathrm{C}_{5} \mathrm{H}_{8} \mathrm{O}_{4}\right]^{-}$; $(-)$ HRESIMS $m / z 1101.5119[\mathrm{M}-\mathrm{H}]^{-}$(calcd for $\left[\mathrm{C}_{53} \mathrm{H}_{81} \mathrm{O}_{24}\right]^{-}, 1101.5123$ ).

Pacificusoside $C(3), \mathrm{C}_{60} \mathrm{H}_{94} \mathrm{O}_{26}$, white amorphous powder; $[\alpha]_{\mathrm{D}}{ }^{25}-9.0(\mathrm{c} 0.23, \mathrm{MeOH})$; IR $(\mathrm{KBr}): v_{\max }=3427,2927,2854 ; 1745,1635,1457,1384,1238,1180,1065 \mathrm{~cm}^{-1} ;{ }^{1} \mathrm{H}$ and ${ }^{13} \mathrm{C} \mathrm{NMR}$ data are listed in Tables 1 and 2; (+)ESIMS/MS of the ion [M+Na ${ }^{+}$at $m / z$ 1253: $1121[(\mathrm{M}+\mathrm{Na})-$ $\left.\mathrm{C}_{5} \mathrm{H}_{8} \mathrm{O}_{4}\right]^{+} ; 759$ [(carbohydrate chain $\left.+\mathrm{Na}\right]^{+} ; 609$ [(carbohydrate chain $\left.\left.+\mathrm{Na}\right)-\mathrm{C}_{5} \mathrm{H}_{8} \mathrm{O}_{4}-\mathrm{H}_{2} \mathrm{O}\right]^{+}$; 477 [(carbohydrate chain $\left.+\mathrm{Na})-2 \times \mathrm{C}_{5} \mathrm{H}_{8} \mathrm{O}_{4}-\mathrm{H}_{2} \mathrm{O}\right]^{+} ; 331$ [(carbohydrate chain $\left.+\mathrm{Na}\right)-2 \times \mathrm{C}_{5} \mathrm{H}_{8} \mathrm{O}_{4}{ }^{-}$ 
$\left.\mathrm{H}_{2} \mathrm{O}-\mathrm{C}_{6} \mathrm{H}_{10} \mathrm{O}_{4}\right]^{+} ; 185\left[\mathrm{C}_{6} \mathrm{H}_{10} \mathrm{O}_{5}+\mathrm{Na}\right]^{+} ; 169\left[\mathrm{C}_{6} \mathrm{H}_{10} \mathrm{O}_{4}+\mathrm{Na}\right]^{+}$; (+)HRESIMS m/z 1253.5920 $[\mathrm{M}+\mathrm{Na}]^{+}$(calcd for $\left.\left[\mathrm{C}_{60} \mathrm{H}_{94} \mathrm{O}_{26} \mathrm{Na}\right]^{+}, 1253.5926\right) ;(-)$ ESIMS/MS of the ion $[\mathrm{M}-\mathrm{H}]^{-}$at $m / z 1229$ : $1097\left[(\mathrm{M}-\mathrm{H})-\mathrm{C}_{5} \mathrm{H}_{8} \mathrm{O}_{4}\right]^{-} ; 1083\left[(\mathrm{M}-\mathrm{H})-\mathrm{C}_{6} \mathrm{H}_{10} \mathrm{O}_{4}\right]^{-} ; 951\left[(\mathrm{M}-\mathrm{H})-\mathrm{C}_{6} \mathrm{H}_{10} \mathrm{O}_{4}-\mathrm{C}_{5} \mathrm{H}_{8} \mathrm{O}_{4}\right]^{-} ; 921[(\mathrm{M}-$ $\left.\mathrm{H})-\mathrm{C}_{6} \mathrm{H}_{10} \mathrm{O}_{4}-\mathrm{C}_{6} \mathrm{H}_{10} \mathrm{O}_{5}\right]^{-} ; 789\left[(\mathrm{M}-\mathrm{H})-\mathrm{C}_{6} \mathrm{H}_{10} \mathrm{O}_{5}-\mathrm{C}_{6} \mathrm{H}_{10} \mathrm{O}_{4}-\mathrm{C}_{5} \mathrm{H}_{8} \mathrm{O}_{4}\right]^{-} ; 643\left[(\mathrm{M}-\mathrm{H})-\mathrm{C}_{6} \mathrm{H}_{10} \mathrm{O}_{5}-\right.$ $\left.2 \times \mathrm{C}_{6} \mathrm{H}_{10} \mathrm{O}_{4}-\mathrm{C}_{5} \mathrm{H}_{8} \mathrm{O}_{4}\right]^{-} ; 511\left[(\mathrm{M}-\mathrm{H})-\mathrm{C}_{6} \mathrm{H}_{10} \mathrm{O}_{5}-2 \times \mathrm{C}_{6} \mathrm{H}_{10} \mathrm{O}_{4}-2 \times \mathrm{C}_{5} \mathrm{H}_{8} \mathrm{O}_{4}\right]^{-}$; (-)HRESIMS $\mathrm{m} / \mathrm{z}$ $1229.5952[\mathrm{M}-\mathrm{H}]^{-}$(calcd for $\left.\left[\mathrm{C}_{60} \mathrm{H}_{93} \mathrm{O}_{26}\right]^{-}, 1229.5961\right)$.

Table $1 .{ }^{1} \mathrm{H}$ and ${ }^{13} \mathrm{C}$ NMR data of aglycon part of compounds $1-3\left(35^{\circ} \mathrm{C}, \mathrm{C}_{5} \mathrm{D}_{5} \mathrm{~N}, \mathrm{~J}\right.$ in $\left.\mathrm{Hz}\right)$ a.

\begin{tabular}{|c|c|c|c|c|c|c|}
\hline \multirow[t]{2}{*}{ Position } & \multicolumn{2}{|c|}{1} & \multicolumn{2}{|c|}{2} & \multicolumn{2}{|c|}{3} \\
\hline & $\delta_{\mathbf{H}}$ & $\delta_{\mathrm{C}}$ & $\delta_{\mathbf{H}}$ & $\delta_{\mathrm{C}}$ & $\delta_{\mathbf{H}}$ & $\delta_{\mathrm{C}}$ \\
\hline 1 & $1.49 \mathrm{~m}$ & 36.3 & $1.48 \mathrm{~m}$ & 35.9 & $1.48 \mathrm{~m}$ & 36.4 \\
\hline 2 & $\begin{array}{l}2.21 \mathrm{~m} \\
1.96 \mathrm{~m}\end{array}$ & 27.2 & $\begin{array}{l}2.16 \mathrm{~m} \\
1.89 \mathrm{~m}\end{array}$ & 26.9 & $\begin{array}{l}2.19 \mathrm{~m} \\
1.94 \mathrm{~m}\end{array}$ & 27.3 \\
\hline 3 & $\begin{array}{c}3.35 \mathrm{dd} \\
(11.6,4.1)\end{array}$ & 89.1 & $\begin{array}{c}3.31 \mathrm{dd} \\
(11.7,3.8)\end{array}$ & 88.9 & $\begin{array}{c}3.34 \mathrm{dd} \\
(11.5,3.9)\end{array}$ & 89.3 \\
\hline 4 & - & 39.7 & - & 39.4 & - & 39.8 \\
\hline 5 & $1.06 \mathrm{~m}$ & 48.1 & $\begin{array}{c}1.00 \mathrm{dd} \\
(11.9,3.6)\end{array}$ & 47.5 & $1.06 \mathrm{~m}$ & 48.3 \\
\hline 6 & $2.08 \mathrm{~m}$ & 23.4 & $\begin{array}{l}2.07 \mathrm{~m} \\
1.97 \mathrm{~m}\end{array}$ & 23.3 & $\begin{array}{l}2.06 \mathrm{~m} \\
1.93 \mathrm{~m}\end{array}$ & 23.6 \\
\hline 7 & $5.68 \mathrm{~m}$ & 121.1 & $\begin{array}{c}5.64 \mathrm{brd} \\
(7.1)\end{array}$ & 122.6 & $5.68 \mathrm{brs}$ & 120.7 \\
\hline 8 & - & 145.3 & - & 147.4 & - & 145.9 \\
\hline 9 & $\begin{array}{c}3.44 \mathrm{brd} \\
(14.2)\end{array}$ & 47.4 & $\begin{array}{c}3.00 \mathrm{brd} \\
(14.6)\end{array}$ & 46.4 & $\begin{array}{c}3.48 \mathrm{brd} \\
(13.5)\end{array}$ & 47.4 \\
\hline 10 & - & 35.8 & - & 35.5 & - & 35.8 \\
\hline 11 & $\begin{array}{l}1.88 \mathrm{~m} \\
1.60 \mathrm{~m}\end{array}$ & 22.5 & $\begin{array}{l}2.00 \mathrm{~m} \\
1.47 \mathrm{~m}\end{array}$ & 21.7 & $\begin{array}{l}1.81 \mathrm{~m} \\
1.55 \mathrm{~m}\end{array}$ & 22.8 \\
\hline 12 & $\begin{array}{l}2.15 \mathrm{~m} \\
2.02 \mathrm{~m}\end{array}$ & 30.7 & $\begin{array}{l}2.37 \mathrm{~m} \\
1.88 \mathrm{~m}\end{array}$ & 20.0 & $\begin{array}{l}2.16 \mathrm{~m} \\
2.00 \mathrm{~m}\end{array}$ & 31.6 \\
\hline 13 & - & 59.1 & - & 56.7 & - & 59.5 \\
\hline 14 & - & 48.2 & - & 46.0 & - & 47.7 \\
\hline 15 & $\begin{array}{c}2.58 \mathrm{dd} \\
(12.5,7.2) \\
1.70 \mathrm{~m}\end{array}$ & 43.3 & $\begin{array}{c}2.16 \mathrm{~d} \\
(13.3) \\
1.97 \mathrm{dd} \\
(13.3,2.2)\end{array}$ & 43.8 & $\begin{array}{c}2.63 \mathrm{dd} \\
(12.4,7.4) \\
1.75 \mathrm{~m}\end{array}$ & 43.9 \\
\hline 16 & $5.81 \mathrm{q}(8.3)$ & 73.6 & $\begin{array}{l}4.74 \text { brd } \\
(2.2)\end{array}$ & 80.4 & 5.96 q (8.7) & 75.2 \\
\hline 17 & $3.01 \mathrm{~d}(8.3)$ & 56.8 & $2.94 \mathrm{~s}$ & 59.1 & $2.66 \mathrm{~d}(9.5)$ & 54.8 \\
\hline 18 & - & 178.5 & - & 180.7 & - & 179.7 \\
\hline 19 & $1.24 \mathrm{~s}$ & 24.1 & $1.02 \mathrm{~s}$ & 23.9 & $1.23 \mathrm{~s}$ & 24.2 \\
\hline 20 & - & 82.4 & - & 139.9 & - & 85.2 \\
\hline 21 & $1.56 \mathrm{~s}$ & 28.9 & $1.75 \mathrm{~s}$ & 23.0 & $1.53 \mathrm{~s}$ & 28.5 \\
\hline 22 & $\begin{array}{l}7.28 \mathrm{~d} \\
(16.0)\end{array}$ & 159.4 & $\begin{array}{l}5.06 \mathrm{~s} \\
4.98 \mathrm{~s}\end{array}$ & 113.9 & $\begin{array}{c}2.57 \mathrm{td} \\
(12.0,4.4) \\
1.93 \mathrm{~m}\end{array}$ & 38.9 \\
\hline 23 & $\begin{array}{c}6.46 \mathrm{dd} \\
(16.0,7.6)\end{array}$ & 127.6 & - & - & $\begin{array}{c}2.07 \mathrm{~m} 2.01 \\
\mathrm{~m}\end{array}$ & 23.6 \\
\hline 24 & $9.78 \mathrm{~d}(7.6)$ & 193.1 & - & - & $5.12 \mathrm{~m}$ & 124.4 \\
\hline 25 & - & - & - & - & - & 132.1 \\
\hline 26 & - & - & - & - & $1.59 \mathrm{~s}$ & 17.9 \\
\hline 27 & - & - & - & - & $1.65 \mathrm{~s}$ & 25.7 \\
\hline 30 & $1.17 \mathrm{~s}$ & 17.6 & $1.12 \mathrm{~s}$ & 17.3 & $1.15 \mathrm{~s}$ & 17.7 \\
\hline 31 & $1.32 \mathrm{~s}$ & 28.7 & $1.30 \mathrm{~s}$ & 28.6 & $1.31 \mathrm{~s}$ & 29.0 \\
\hline 32 & $1.15 \mathrm{~s}$ & 32.3 & $1.35 \mathrm{~s}$ & 33.9 & $1.17 \mathrm{~s}$ & 32.4 \\
\hline $\mathrm{CO}$ & - & 169.7 & - & - & - & 170.0 \\
\hline $\mathrm{CH}_{3}-\mathrm{CO}$ & $1.86 \mathrm{~s}$ & 21.1 & - & - & $2.01 \mathrm{~s}$ & 21.2 \\
\hline
\end{tabular}

a Assignments from $700 \mathrm{MHz}^{1} \mathrm{H}^{1}{ }^{1} \mathrm{H}$ COSY, HSQC, HMBC (8 Hz), and ROESY (270 msec) data. 
Table 2. ${ }^{1} \mathrm{H},{ }^{13} \mathrm{C}, \mathrm{HMBC}$, and ROESY NMR data of oligosaccharide chains of $1-3\left(35^{\circ} \mathrm{C}, \mathrm{C}_{5} \mathrm{D}_{5} \mathrm{~N}\right.$, $J$ in $\mathrm{Hz})^{\text {a }}$.

\begin{tabular}{|c|c|c|c|c|}
\hline \multirow{2}{*}{ Position } & \multicolumn{4}{|c|}{$1-3$} \\
\hline & $\delta_{\mathrm{H}}$ & $\delta_{\mathrm{C}}$ & HMBC & ROESY \\
\hline & \multicolumn{4}{|c|}{$X y l-I$} \\
\hline 1 & $4.84 \mathrm{~d}(7.1)$ & 105.0 & C3-Agl; C5-Xyl-I & $\begin{array}{l}\text { H3, H30-Agl; } \\
\text { H3, H5-Xyl-I }\end{array}$ \\
\hline 2 & $3.91 \mathrm{dd}(8.7,7.1)$ & 83.2 & $\begin{array}{l}\text { C1, C3-Xyl-I; } \\
\text { C1-Qui }\end{array}$ & H4-Xyl-I \\
\hline 3 & $4.19 \mathrm{t}(8.7)$ & 77.8 & C2, C4-Xyl-I & H1-Xyl-I \\
\hline 4 & $4.11 \mathrm{~m}$ & 70.2 & C3-Xyl-I & H2-Xyl-I \\
\hline \multirow[t]{2}{*}{5} & $\begin{array}{l}4.32 \mathrm{dd}(11.6,5.4) \\
3.69 \mathrm{dd}(11.6,9.3)\end{array}$ & 66.5 & $\begin{array}{l}\text { C1, C3-Xyl-I } \\
\text { C1, C3-Xyl-I }\end{array}$ & H1-Xyl-I \\
\hline & \multicolumn{4}{|l|}{ 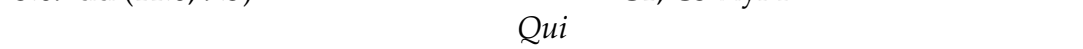 } \\
\hline 1 & $5.20 \mathrm{~d}(7.3)$ & 103.0 & C2-Xyl-I & $\begin{array}{l}\text { H3, H5-Qui; } \\
\text { H2-Xyl-I }\end{array}$ \\
\hline 2 & $4.11 \mathrm{~m}$ & 82.7 & $\begin{array}{l}\text { C1, C3-Qui; } \\
\text { C1-Xyl-II }\end{array}$ & \\
\hline 3 & $4.09 \mathrm{~m}$ & 75.6 & C2, C4-Qui & H1-Qui \\
\hline 4 & $3.61 \mathrm{~m}$ & 86.5 & $\begin{array}{c}\text { C3, C5, C6-Qui; } \\
\text { C1-Glc }\end{array}$ & H1-Glc; H6-Qui \\
\hline 5 & $3.67 \mathrm{~m}$ & 71.0 & C4-Qui & H1-Qui \\
\hline 6 & \multicolumn{4}{|c|}{ 年 } \\
\hline 1 & $4.94 \mathrm{~d}(7.9)$ & 104.7 & C4-Qui & $\begin{array}{l}\text { H4-Qui; H3, } \\
\text { H5-Glc }\end{array}$ \\
\hline 2 & $4.00 \mathrm{~m}$ & 73.8 & C1, C3-Glc & \\
\hline 3 & $4.21 \mathrm{t}(9.0)$ & 87.1 & C2, C4-Glc & $\begin{array}{c}\text { H1-3-OMe-Xyl; } \\
\text { H1, H5-Glc }\end{array}$ \\
\hline $\begin{array}{l}4 \\
5\end{array}$ & $\begin{array}{c}4.05 \mathrm{t}(9.0) \\
3.99 \mathrm{~m}\end{array}$ & $\begin{array}{l}69.3 \\
77.8\end{array}$ & C3, C5, C6-Glc & \\
\hline \multirow[t]{2}{*}{6} & $\begin{array}{c}3.99 \mathrm{~m} \\
4.47 \mathrm{dd}(11.5,2.6) \\
4.20 \mathrm{~m}\end{array}$ & $\begin{array}{l}77.8 \\
61.7\end{array}$ & & H1, H3-Glc \\
\hline & \multicolumn{4}{|c|}{ 3-OMe-Xyl } \\
\hline 1 & $5.21 \mathrm{~d}(7.6)$ & 106.0 & $\begin{array}{c}\text { C3-Glc; } \\
\text { C5-3-OMe-Xyl }\end{array}$ & $\begin{array}{c}\text { H3, } \\
\text { H5-3-OMeXyl; } \\
\text { H3-Glc }\end{array}$ \\
\hline 2 & 3.94 t (8.4) & 74.6 & $\begin{array}{c}\text { C1, } \\
\text { C3-3-OMe-Xyl }\end{array}$ & H4-3-OMe-Xyl \\
\hline 3 & 3.60 t (8.8) & 87.7 & $\begin{array}{c}\text { C2, } \\
\text { C4-3-OMe-Xyl; } \\
\text { OMe }\end{array}$ & $\begin{array}{c}\text { H1-3-OMe-Xyl; } \\
\text { OMe }\end{array}$ \\
\hline 4 & $4.08 \mathrm{~m}$ & 69.9 & $\mathrm{C} 1$ & H2-3-OMe-Xyl \\
\hline 5 & $\begin{array}{c}4.21 \mathrm{dd}(11.3,5.5) \\
\quad 3.63 \mathrm{t}(11.3)\end{array}$ & 67.0 & $\begin{array}{c}\text { C3-3-OMe-Xyl } \\
\text { C1, C3, } \\
\text { C4-3-OMe-Xyl }\end{array}$ & H1-3-OMe-Xyl \\
\hline 3-OMe & \multicolumn{3}{|c|}{ Xyl-II } & H3-3-OMe-Xyl \\
\hline 1 & $5.36 \mathrm{~d}(7.0)$ & 105.8 & $\begin{array}{l}\text { C2-Qui; } \\
\text { C5-Xyl-II }\end{array}$ & $\begin{array}{l}\text { H3, H5-Xyl-II; } \\
\text { H2-Qui }\end{array}$ \\
\hline 2 & $4.04 \mathrm{~m}$ & 75.5 & C1, C3-Xyl-II & \\
\hline 3 & $4.10 \mathrm{t}(8.1)$ & 77.0 & C2, C4-Xyl-II & H1-Xyl-II \\
\hline 4 & $4.12 \mathrm{~m}$ & 70.4 & C3-Xyl-II & \\
\hline 5 & $\begin{array}{l}4.33 \mathrm{dd}(11.7,4.6) \\
3.65 \mathrm{dd}(11.7,9.6)\end{array}$ & 66.9 & $\begin{array}{l}\text { C1, C3, C4-Xyl-II } \\
\text { C1, C4, C5-Xyl-II }\end{array}$ & H1-Xyl-II \\
\hline
\end{tabular}

a Assignments from $700 \mathrm{MHz}{ }^{1} \mathrm{H}^{-1} \mathrm{H}$ COSY, HSQC, HMBC (8 Hz), and ROESY (270 msec) data. 


\subsection{Acid Hydrolysis and Determination of Absolute Configurations of Monosaccharides}

The acid hydrolysis of $\mathbf{1}(3.5 \mathrm{mg})$ was carried out in a solution of $2 \mathrm{M}$ trifluoroacetic acid (TFA) $(1 \mathrm{~mL})$ in a sealed vial on a $\mathrm{H}_{2} \mathrm{O}$ bath at $100{ }^{\circ} \mathrm{C}$ for $2 \mathrm{~h}$. The $\mathrm{H}_{2} \mathrm{O}$ layer was washed with $\mathrm{CHCl}_{3}(3 \times 1.0 \mathrm{~mL})$ and concentrated in vacuo. One drop of concentrated TFA and $0.5 \mathrm{~mL}$ of L-(-)-2-octanol (Sigma Aldrich) were added to the sugar mixture, and the sealed vial was heated on a glycerol bath at $130^{\circ} \mathrm{C}$ for $6 \mathrm{~h}$. The solution was evaporated in vacuo and treated with a mixture of pyridine/acetic anhydride (1:1, $0.6 \mathrm{~mL})$ for $24 \mathrm{~h}$ at room temperature. The acetylated 2-octylglycosides were analyzed by GC using the corresponding authentic samples prepared by the same procedure. The following peaks were detected in the hydrolysate of 1 : D-quinovose $\left(t_{R} 20.32,20.55,21.91\right.$, and $\left.21.13 \mathrm{~min}\right)$, D-xylose $\left(t_{\mathrm{R}} 20.75,20.91\right.$, and $\left.21.19 \mathrm{~min}\right)$, D-glucose ( $t_{\mathrm{R}} 24.24,24.86,25.10$, and $\left.25.38 \mathrm{~min}\right)$, and 3-OMe-D-xylose $\left(t_{\mathrm{R}} 19.33,19.57,19.84\right.$, and $\left.20.00 \mathrm{~min}\right)$. The retention times of the authentic samples were as follows: D-quinovose $\left(t_{R} 20.30,20.50,20.86\right.$, and $\left.21.13 \mathrm{~min}\right)$, D-xylose ( $t_{\mathrm{R}} 20.70,20.89$, and $\left.21.15 \mathrm{~min}\right)$, D-glucose $\left(t_{\mathrm{R}} 24.23,24.83,25.06\right.$, and $\left.25.37 \mathrm{~min}\right)$, 3-OMe-D-xylose $\left(t_{\mathrm{R}} 19.32,19.57,19.86\right.$, and $\left.20.02 \mathrm{~min}\right)$, L-quinovose $\left(t_{\mathrm{R}} 20.12,20.60\right.$, and $21.17 \mathrm{~min})$, L-xylose ( $t_{\mathrm{R}} 20.57,21.03$, and $\left.21.34 \mathrm{~min}\right)$, L-glucose $\left(t_{\mathrm{R}} 24.39,24.63,24.83\right.$, and $25.06 \mathrm{~min})$, and 3-OMe-L-xylose ( $t_{\mathrm{R}} 18.92,19.50$, and $\left.20.10 \mathrm{~min}\right)$.

\subsection{Anticancer Activity}

\subsubsection{Reagents}

Phosphate buffered saline (PBS), L-glutamine, penicillin-streptomycin solution $(10,000 \mathrm{U} / \mathrm{mL}, 10 \mu \mathrm{g} / \mathrm{mL}$ ), dimethyl sulfoxide (DMSO), $\mathrm{BuOH}, 99.9 \%$ (pure p.a.), and cholesterol, 99.5\% were from Sigma-Aldrich. MTS reagent (3-(4,5-dimethylthiazol-2yl)-5-(3-carboxymethoxyphenyl)-2-(4-sulfophenyl)-2H-tetrazolium) was purchased from Promega (Madison, WI, USA). The Basal Medium Eagle (BME), Minimum Essential Medium Eagle (MEM), Dulbecco's Modified Eagle's Medium (DMEM), McCoy's 5A Modified Medium (McCoy's 5A), trypsin, fetal bovine serum (FBS), and agar were purchased from Thermo Fisher Scientific (Waltham, MA, USA). Organic solvents, inorganic acids, and salts were commercial products (Laverna, Moscow, Russia).

\subsubsection{Cell Lines and Culture Conditions}

Human embryonic kidney HEK 293 cells (ATCC ${ }^{\circledR}$ no. CRL-1573 ${ }^{\mathrm{TM}}$ ), colorectal carcinoma HT-29 cells (ATCC ${ }^{\circledR}$ no. HTB-38 ${ }^{\mathrm{TM}}$ ), melanoma RPMI-7951 cells (ATCC ${ }^{\circledR}$ no. HTB-66), and breast cancer MDA-MB-231 cells (ATCC ${ }^{\circledR} \mathrm{HTB}-26^{\mathrm{TM}}$ ) were obtained from the American Type Culture Collection (Manassas, VA, USA). HEK 293, HT-29, RPMI-7951, and MDA-MB-231cells were cultured in complete MEM/10\% FBS, McCoy's 5A/10\% FBS, and DMEM $/ 10 \%$ FBS, respectively, contained $1 \%$ of penicillin-streptomycin solution. The cell cultures were maintained at $37{ }^{\circ} \mathrm{C}$ in humidified atmosphere containing $5 \% \mathrm{CO}_{2}$. Every 3-4 days cells were rinsed with PBS, detached from the tissue culture flask by $0.25 \%$ trypsin $/ 0.05 \mathrm{M}$ EDTA, and $10-20 \%$ of the harvested cells were transferred to a new flask containing fresh culture media. After three passages the cells were used for the experiments.

\subsubsection{The Preparation of Solutions of Compounds}

Compounds 1-6 were dissolved in sterile DMSO to prepare stock concentrations of $20 \mathrm{mM}$. Cells were treated with serially diluted compounds 1-6 (culture medium used as diluent) to give the intended final concentrations. The vehicle control is the cells treated with equivalent volume of DMSO (less than $0.5 \%$ ) for all presented experiments.

Cholesterol $(10 \mathrm{mg} / \mathrm{mL})$ was dissolved in $99.5 \% \mathrm{BuOH}$ and added to compounds 1-6 to get their stock concentrations of $20 \mathrm{mM}$ and incubated for $12 \mathrm{~h}$ at room temperature. Then samples were vacuum evaporated and dissolved in DMSO to their stock concentrations of $20 \mathrm{mM}$. 


\subsubsection{Cell Viability Assay}

Cells $\left(1.0 \times 10^{4}\right)$ were seeded in 96-well plates (Jet Biofil, Guangzhou, China) and cultured in $200 \mu \mathrm{L}$ of complete culture medium for $24 \mathrm{~h}$ at $37^{\circ} \mathrm{C}$ in $5 \% \mathrm{CO}_{2}$ incubator. The cell monolayer was treated with compounds 1-6 or 1-6 combined with cholesterol at concentrations of 5, 10,20, and $40 \mu \mathrm{M}$ in fresh appropriate culture medium for $24 \mathrm{~h}$. Cells were also treated by cisplatin (positive control) at 1, 5, 10, 50, $100 \mu \mathrm{M}$ for 24 and $48 \mathrm{~h}$. Subsequently, the cells were incubated with $15 \mu \mathrm{L}$ MTS reagent for $3 \mathrm{~h}$, and the absorbance of each well was measured at 490/630 nm using Power Wave XS microplate reader (BioTek, Wynusky, VT, USA).

\subsubsection{Colony Formation Assay}

Human cancer cells $\left(2.4 \times 10^{4} / \mathrm{mL}\right)$ were seeded into 6-well plate and treated with compounds 1-6 $(0.1,0.5$, and $1 \mu \mathrm{M})$ or cisplatin $(3 \mu \mathrm{M})$ in $1 \mathrm{~mL}$ of $0.3 \%$ BME agar containing $10 \%$ FBS, $2 \mathrm{mM}$ L-glutamine, and $25 \mu \mathrm{g} / \mathrm{mL}$ gentamicin. The cultures were maintained in a $37{ }^{\circ} \mathrm{C}, 5 \% \mathrm{CO}_{2}$ incubator for 14 days, and the cell colonies were scored using a Motic microscope AE 20 (XiangAn, Xiamen, China) and ImageJ software bundled with 64-bit Java 1.8.0_112 (NIH, Bethesda, MD, USA).

\subsubsection{Statistical Analysis}

All assays were performed in at least three independent experiments. Results are expressed as the mean \pm standard deviation (SD). Student's $t$ test was used to evaluate the data with the following significance levels: ${ }^{*} p<0.05,{ }^{* *} p<0.01,{ }^{* * *} p<0.001$.

\section{Results}

\subsection{The Isolation and Structure Elucidation of Compounds 1-6 from S. pacificus}

The concentrated ethanol extract of $S$. pacificus was subjected to sequential separation by chromatography on columns with Polichrom-1, Si gel, and Florisil followed by HPLC on semipreparative Diasfer-110-C18 and Discovery $\mathrm{C}_{18}$ columns to yield three new triterpene glycosides, named as pacificusosides $\mathrm{A}-\mathrm{C}(\mathbf{1}-\mathbf{3})$, and three previously known triterpene glycosides 4-6 (Figure 1). Compounds 4-6 were identified by comparison of their ${ }^{1} \mathrm{H}$ and ${ }^{13} \mathrm{C}$ NMR, and MS spectra with those reported for cucumariosides $C_{1}(4), C_{2}(5)$, and $A_{10}$ (6), triterpene glycosides from the holothurian Eupentacta fraudatrix [22,23].

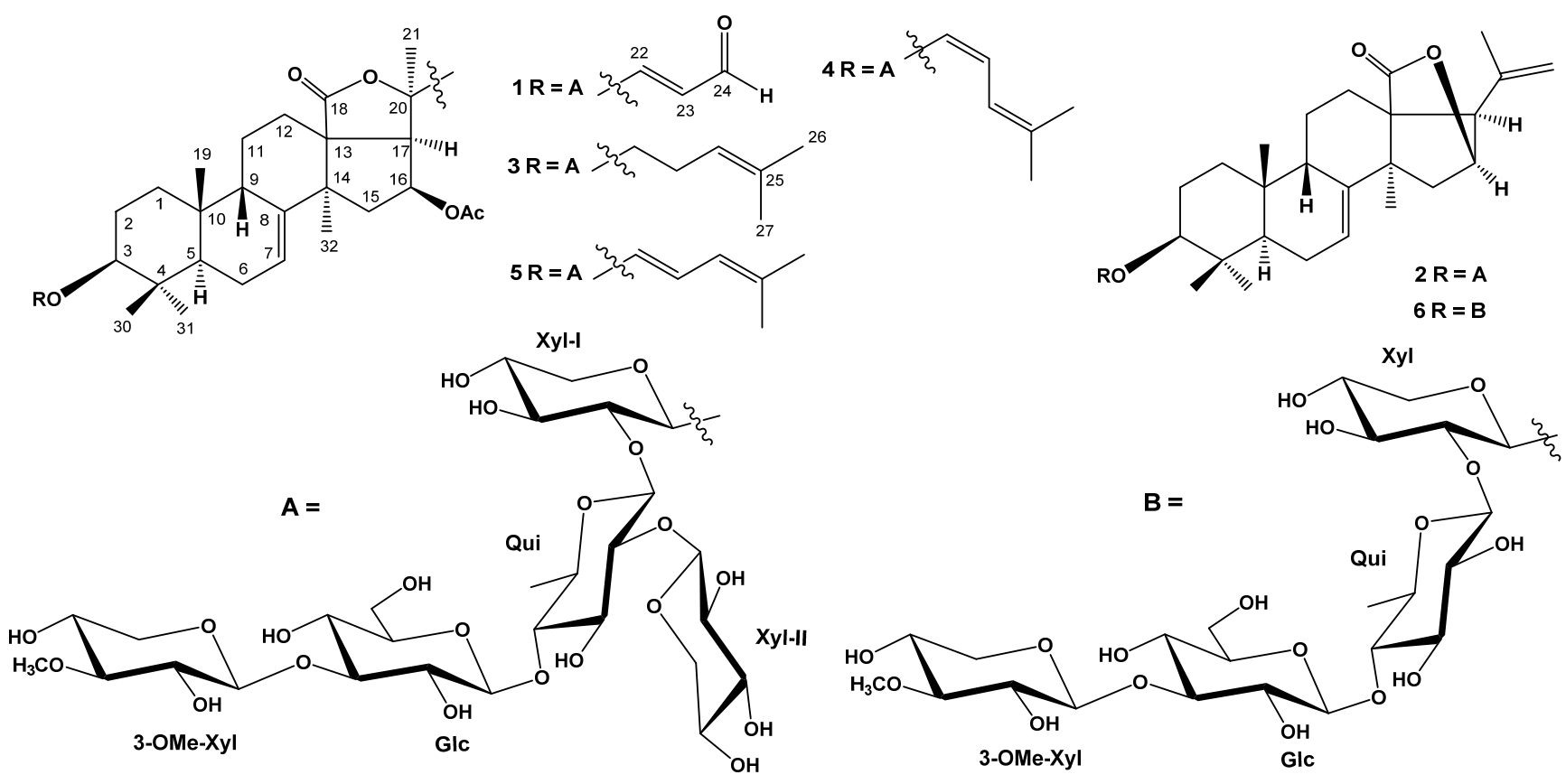

Figure 1. The structures of compounds 1-6 isolated from S. pacificus. 
The molecular formula of $\mathbf{1}$ was determined to be $\mathrm{C}_{57} \mathrm{H}_{86} \mathrm{O}_{27}$ from the [M+Na] $]^{+}$ sodiated molecular ion peak at $m / z 1225.5273$ (calcd for $\left[\mathrm{C}_{57} \mathrm{H}_{86} \mathrm{O}_{27} \mathrm{Na}\right]^{+}, 1225.5249$ ) in the (+)HRESIMS and from the $[\mathrm{M}-\mathrm{H}]^{-}$ion peak at $m / z 1201.5318\left(\right.$ calcd for $\left[\mathrm{C}_{57} \mathrm{H}_{85} \mathrm{O}_{27}\right]^{-}$, 1201.5284) in the (-)HRESIMS (Figure S1). The IR spectrum of compound 1 showed the presence of hydroxy group $\left(3438 \mathrm{~cm}^{-1}\right)$, lactone carbonyl $\left(1745 \mathrm{~cm}^{-1}\right)$ and olefinic $\left(1633 \mathrm{~cm}^{-1}\right)$ groups, as well as one characteristic absorption band at $v_{\max }=1688 \mathrm{~cm}^{-1}$ of $\alpha, \beta$-unsaturated aldehyde group (Figure S2). The ${ }^{1} \mathrm{H}$ and ${ }^{13} \mathrm{C}$ NMR spectroscopic data of the pentacyclic moiety of the aglycon of 1 showed the resonances of protons and carbons of four methyl groups $\left(\delta_{\mathrm{H}} 1.15 \mathrm{~s}, 1.17 \mathrm{~s}, 1.24 \mathrm{~s}, 1.32 \mathrm{~s} ; \delta_{\mathrm{C}} 32.3,17.6,24.1,28.7\right)$, the 7(8)-double bond $\left(\delta_{\mathrm{H}} 5.68 \mathrm{~m} ; \delta_{\mathrm{C}} 121.1,145.3\right)$, two oxygenated methine groups $\left[\delta_{\mathrm{H}} 3.35 \mathrm{dd}(J=11.6\right.$, $\left.4.1), 5.81 \mathrm{q}(J=8.3) ; \delta_{\mathrm{C}} 89.1,73.6\right]$, one OAc-group $\left(\delta_{\mathrm{H}} 1.86 \mathrm{~s}, \delta_{\mathrm{C}} 21.1,169.7\right)$, and one lactone carbonyl $\left(\delta_{\mathrm{C}}\right.$ 178.5) (Table 1, Figures S4 and S5). The ${ }^{1} \mathrm{H}^{-1} \mathrm{H}$ COSY and HSQC correlations attributable to triterpene nucleus revealed the corresponding sequences of protons from C-1 to C-3, C-5 to C-7, C-9 to C-12 through C-11, and C-15 to C-17 (Figure 2A, Figures S6 and S7). Key HMBC cross-peaks, such as H-3/C-30; H-5/C-10, C-19; H-6/C-10; H-7/C-9; H-12/C-13, C-14, C-18; H-15/C-13, C-14, C-17, C-32; H-16/CO(OAc); H-17/C-18, $\mathrm{C}-21 ; \mathrm{H}_{3}-19 / \mathrm{C}-1, \mathrm{C}-5, \mathrm{C}-9, \mathrm{C}-10 ; \mathrm{H}_{3}-30 / \mathrm{C}-3, \mathrm{C}-4, \mathrm{C}-5, \mathrm{C}-31 ; \mathrm{H}_{3}-31 / \mathrm{C}-3, \mathrm{C}-4, \mathrm{C}-5, \mathrm{C}-30 ; \mathrm{H}_{3}-$ $32 / \mathrm{C}-8, \mathrm{C}-14, \mathrm{C}-15 ; \mathrm{H}_{3} \mathrm{C}(\mathrm{OAc}) / \mathrm{CO}(\mathrm{OAc})$ confirmed the overall structure of the pentacyclic triterpene moiety of 1 (Figure 2A, Figure S8). The key ROESY cross-peaks $\mathrm{H}-1 \alpha / \mathrm{H}-3, \mathrm{H}-5 \alpha$; $\mathrm{H}-2 \beta / \mathrm{H}_{3}-19, \mathrm{H}_{3}-30 ; \mathrm{H}-3 / \mathrm{H}-5 \alpha ; \mathrm{H}-5 \alpha / \mathrm{H}_{3}-31 ; \mathrm{H}-6 \beta / \mathrm{H}_{3}-19 ; \mathrm{H}-7 / \mathrm{H}-15 \alpha ; \mathrm{H}-9 \beta / \mathrm{H}_{3}-19 ; \mathrm{H}-$ $12 \alpha / \mathrm{H}_{3}-21, \mathrm{H}_{3}-32 ; \mathrm{H}-15 \alpha / \mathrm{H}_{3}-32 ; \mathrm{H}-16 \alpha / \mathrm{H}_{3}-32 ; \mathrm{H}-17 \alpha / \mathrm{H}_{3}-21, \mathrm{H}_{3}-32$ showed the common $5 \alpha / 9 \beta / 10 \beta / 13 \beta, 14 \alpha$ stereochemistry of the triterpene nucleus and $3 \beta, 16 \beta$-configurations of oxygenated substituents in $\mathbf{1}$ (Figure 2B, Figure S9).
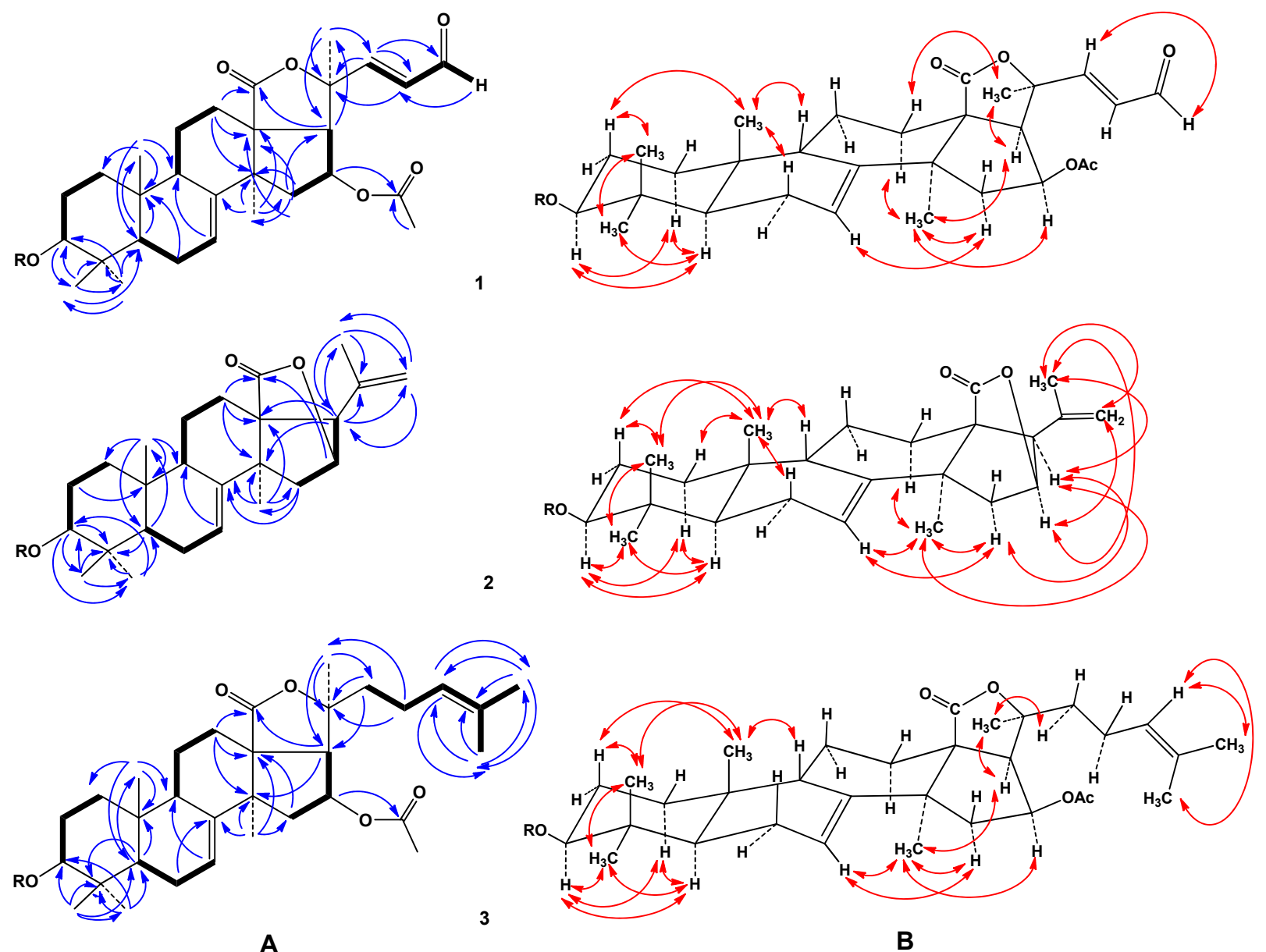

Figure 2. (A) ${ }^{1} \mathrm{H}_{-}{ }^{1} \mathrm{H}$ COSY and key HMBC correlations for aglycon part of compounds 1-3. (B) Key ROESY correlations for aglycon part of compounds $\mathbf{1}-\mathbf{3}$. 
Table 1 demonstrated the presence of one methyl group $\left(\delta_{\mathrm{H}} 1.56 \mathrm{~s} ; \delta_{\mathrm{C}} 28.9\right)$ and $\alpha, \beta-$ unsaturated aldehyde group $\left[\delta_{\mathrm{H}} 7.28 \mathrm{~d}(J=16.0), 6.46 \mathrm{dd}(J=16.0,7.6), 9.78 \mathrm{~d}(7.6) ; \delta_{\mathrm{C}} 159.4\right.$, 127.6, 193.1] (Table 1, Figures S4 and S5). The existence of the $\alpha, \beta$-unsaturated aldehyde group was confirmed by the UV spectrum $\left(\lambda_{\max }=224 \mathrm{~nm}\right.$ in $\left.\mathrm{MeOH}\right)$ (Figure S3). The protons sequence from $\mathrm{H}-22$ to $\mathrm{H}-24$, correlated with the corresponding carbon atoms of the side chain of 1, was assigned using the COSY and HSQC experiments (Table 1, Figures S6 and S7). The key HMBC correlations $\mathrm{H}_{3}-21 / \mathrm{C}-17, \mathrm{C}-20, \mathrm{C}-22 ; \mathrm{H}-22$ /C-20, C23, C-24; H-23/C-20; and H-24/C-23 and ROESY correlation H-22/H-24 supported the total structure of the $\Delta^{22}$-24-aldo-25,26,27-trinorlanostane side chain (Figure 1 ). The trans configuration of 22(23)-double bond followed from $J_{22,23}=16.0 \mathrm{~Hz}$. To the best of our knowledge, compound 1 has a novel type of triterpene aglycon, containing $\Delta^{22}-25,26,27$ trinorlanostane side chain with unique C-24 aldehyde group, never found before in the sea cucumber triterpene glycosides or starfish steroidal glycosides.

In addition to the above-mentioned signals, the ${ }^{1} \mathrm{H}$ NMR spectrum of 1 exhibited five resonances in the deshielded region due to the anomeric protons of monosaccharide units at $\delta_{\mathrm{H}} 4.84,5.20,4.94,5.21$, and 5.36 that correlated in the HSQC experiment with a carbon signals at $\delta_{\mathrm{C}} 105.0,103.0,104.7,106.0$, and 105.8, respectively, as well as one resonance due to an $O$-methyl group at $\delta_{\mathrm{H}} 3.85$ that correlated in the HSQC experiment with a carbon signal at $\delta_{\mathrm{C}} 60.4$ (Table 2, Figures S4 and S5).

Along with mass spectral information, these data showed the presence of five monosaccharide residues in the oligosaccharide moiety of 1 . The existence of 6-deoxy-sugar unit was supported by methyl doublet at $\delta_{\mathrm{H}} 1.70$. The coupling constants $(7.0-8.0 \mathrm{~Hz})$ of the anomeric protons were indicative of $\beta$-configurations of all glycosidic bonds. The chemical shifts and coupling constants of $\mathrm{H}-1^{\prime}-\mathrm{H}-6^{\prime}$ of the monosaccharide units were determined by irradiation of anomeric protons in the 1D TOCSY experiment. The ${ }^{1} \mathrm{H}-{ }^{1} \mathrm{H}$ COSY, HSQC, $\mathrm{HMBC}$, and ROESY experiments led to the assignment of all the proton and carbon signals of the carbohydrate chain of 1 (Table 2, Figures S4-S9). The spectral data of the oligosaccharide moiety strictly coincided with those of terminal 3-O-methyl- $\beta$-D-xylopyranosyl and $\beta$-D-xylopyranosyl residues, and internal 3-O-substituted $\beta$-D-glucopyranosyl, 2,4-di-Osubstituted $\beta$-D-quinovopyranosyl, and 2-O-substituted $\beta$-D-xylopyranosyl residues as in the earlier reported spectra of known cucumarioside $\mathrm{C}_{1}$ from E. fraudatrix [22].

On the basis of all the above-mentioned data, the structure of pacificusoside $A(1)$ was determined as 3 $\beta$-O-\{3-O-methyl- $\beta$-D-xylopyranosyl- $(1 \rightarrow 3)-\beta-\mathrm{D}-\mathrm{glucopyranosyl-}(1 \rightarrow 4)-[\beta-\mathrm{D}-$ xylopyranosyl-( $1 \rightarrow 2)]-\beta$-D-quinovopyranosyl- $(1 \rightarrow 2)-\beta$-D-xylopyranosyl $\}-16 \beta$-acetoxy-25,26,27trinorholosta-7,22-dien-24-al.

The molecular formula of 2 was determined to be $\mathrm{C}_{53} \mathrm{H}_{82} \mathrm{O}_{24}$ from the $[\mathrm{M}+\mathrm{Na}]^{+}$ sodiated-molecular ion peak at $m / z 1125.5091$ (calcd for $\left[\mathrm{C}_{53} \mathrm{H}_{82} \mathrm{O}_{24} \mathrm{Na}\right]^{+}, 1125.5088$ ) in the (+)HRESIMS and from the $[\mathrm{M}-\mathrm{H}]^{-}$ion peak at $\mathrm{m} / z 1101.5119$ (calcd for $\left[\mathrm{C}_{53} \mathrm{H}_{81} \mathrm{O}_{24}\right]^{-}$, 1101.5123) in the (-)HRESIMS (Figure S10). The IR spectrum of compound 2 showed the presence of hydroxy group $\left(3427 \mathrm{~cm}^{-1}\right)$, lactone carbonyl $\left(1772 \mathrm{~cm}^{-1}\right)$, and olefinic $\left(1635 \mathrm{~cm}^{-1}\right.$ ) groups (Figure S11). The ${ }^{1} \mathrm{H}$ and ${ }^{13} \mathrm{C}$ NMR spectroscopic data belonging to the pentacyclic moiety of the aglycon of $\mathbf{2}$ showed the resonances of the protons and carbons of four methyl groups $\left(\delta_{\mathrm{H}} 1.02 \mathrm{~s}, 1.12 \mathrm{~s}, 1.30 \mathrm{~s}, 1.35 \mathrm{~s} ; \delta_{\mathrm{C}} 23.9,17.3,28.6,33.9\right)$, a $7(8)$-double bond $\left[\delta_{\mathrm{H}} 5.64\right.$ brd $\left.(7.1) ; \delta_{\mathrm{C}} 122.6,147.4\right)$, two oxygenated methine groups $\left[\delta_{\mathrm{H}} 3.31 \mathrm{dd}(J=11.7,3.8), 4.74\right.$ brd $\left.(J=2.2) ; \delta_{\mathrm{C}} 88.9,80.4\right]$, and one lactone carbonyl $\left(\delta_{\mathrm{C}}\right.$ 180.7) (Table 1, Figures S12 and S13). The proton and carbon signals of the OAc-group in the ${ }^{1} \mathrm{H}$ and ${ }^{13} \mathrm{C}$ NMR spectra of compound 2 were absent. The ${ }^{1} \mathrm{H}^{1}{ }^{1} \mathrm{H}$ COSY and HSQC correlations attributable to triterpene nucleus revealed the corresponding sequences of protons from C-1 to C-3, C-5 to C-7, C-9 to C-12 through C-11, and C-15 to C-17 (Figure 2A, Figures S14 and S15). Key HMBC cross-peaks, such as H-2/C-10; H-3/C-4, C-30, C-31; H-5/C-4, C-19; H-7/C-9; H-12/C-13, C-14, C-18; H-15/C-8, C-32; H-16/C-18; H-17/C13, C-14, C-20, C21, C-22; $\mathrm{H}_{3}-19 / \mathrm{C}-1, \mathrm{C}-5, \mathrm{C}-9, \mathrm{C}-10 ; \mathrm{H}_{3}-30 / \mathrm{C}-3, \mathrm{C}-4, \mathrm{C}-31 ; \mathrm{H}_{3}-31 / \mathrm{C}-4$, $\mathrm{C}-5, \mathrm{C}-30 ; \mathrm{H}_{3}-32 / \mathrm{C}-8, \mathrm{C}-13, \mathrm{C}-14, \mathrm{C}-15$ confirmed the overall structure of the pentacyclic triterpene moiety of 2 (Figure 2A, Figure S16). The key ROESY cross-peaks H-1 $\alpha / \mathrm{H}-3$, 
H- $5 \alpha$; H-2 $\beta / \mathrm{H}_{3}-19, \mathrm{H}_{3}-30 ; \mathrm{H}-3 / \mathrm{H}-5 \alpha, \mathrm{H}_{3}-31 ; \mathrm{H}-5 \alpha / \mathrm{H}_{3}-31 ; \mathrm{H}-6 \beta / \mathrm{H}_{3}-19 ; \mathrm{H}-7 / \mathrm{H}-15 \alpha, \mathrm{H}_{3}-$ $32 ; \mathrm{H}-9 \beta / \mathrm{H}_{3}-19 ; \mathrm{H}-12 \alpha / \mathrm{H}_{3}-32 ; \mathrm{H}-15 \alpha / \mathrm{H}-17 \alpha, \mathrm{H}_{3}-32 ; \mathrm{H}-17 \alpha / \mathrm{H}_{3}-32$ showed the common $5 \alpha / 9 \beta / 10 \beta / 13 \beta, 14 \alpha$ stereochemistry of the triterpene nucleus and $3 \beta, 16 \beta$-configurations of oxygenated substituents in 2 (Figure 2B, Figure S17). The proton and carbon signals of the aglycon side chain of 2 demonstrated the presence of only one methyl group $\left(\delta_{\mathrm{H}}\right.$ $\left.1.75 \mathrm{~s} ; \delta_{\mathrm{C}} 23.0\right)$ and one 20,22-double bond $\left(\delta_{\mathrm{H}} 5.06 \mathrm{~s}, 4.98 \mathrm{~s} ; \delta_{\mathrm{C}} 139.9,113.9\right)$. The key HMBC correlations $\mathrm{H}_{3}-21 / \mathrm{C}-17, \mathrm{C}-20, \mathrm{C}-22$ and H-22/C-17, C-21 and the key ROESY correlations $\mathrm{H}_{3}-21 / \mathrm{H}-16, \mathrm{H}-17, \mathrm{H}-22$; and $\mathrm{H}_{2}-22 / \mathrm{H}-16$ supported the total structure of the side chain (Figure 2A,B). The NMR spectroscopic data of aglycon part of 2 were coincident with those for known cucumariosides $\mathrm{A}_{10}$ and $\mathrm{I}_{4}$ from the sea cucumber $E$. fraudatrix with $23,24,25,26,27-$ pentanorlanosta-7,20(22)-diene-18(16)-lactone-3 $\beta$-ol aglycon [23,24], but the carbohydrate chain was different.

On the basis of extensive 2D NMR and MS analysis of glycosides 1-3, we have suggested that oligosaccharide moiety of $\mathbf{1}$ are identical to those of glycosides $\mathbf{2}$ and $\mathbf{3}$. On the basis of all the above-mentioned data, the structure of pacificusoside B (2) was elucidated to be $3 \beta-O-\{3-O$-methyl- $\beta$-D-xylopyranosyl- $(1 \rightarrow 3)-\beta$-D-glucopyranosyl- $(1 \rightarrow 4)-[\beta$-Dxylopyranosyl-( $(1 \rightarrow 2)]-\beta$-D-quinovopyranosyl-( $1 \rightarrow 2)-\beta$-D-xylopyranosyl $\}-23,24,25,26,27-$ pentanorlanosta-7,20(22)-diene-18(16)-lactone.

The molecular formula of 3 was determined to be $\mathrm{C}_{60} \mathrm{H}_{94} \mathrm{O}_{26}$ from the $[\mathrm{M}+\mathrm{Na}]^{+}$sodiatedmolecular ion peak at $m / z 1253.5920$ (calcd for $\left[\mathrm{C}_{60} \mathrm{H}_{94} \mathrm{O}_{26} \mathrm{Na}\right]^{+}, 1253.5926$ ) in the (+)HRESIMS and from the $[\mathrm{M}-\mathrm{H}]^{-}$ion peak at $\mathrm{m} / \mathrm{z} 1229.5952$ (calcd for $\left[\mathrm{C}_{60} \mathrm{H}_{93} \mathrm{O}_{26}\right]^{-}, 1229.5961$ ) in the (-)HRESIMS (Figure S18). The IR spectrum of compound 3 showed the presence of hydroxy $\left(3427 \mathrm{~cm}^{-1}\right)$, lactone carbonyl $\left(1745 \mathrm{~cm}^{-1}\right)$, and olefinic $\left(1635 \mathrm{~cm}^{-1}\right)$ groups (Figure S19). The thorough comparison of the ${ }^{1} \mathrm{H}$ and ${ }^{13} \mathrm{C}$ NMR data of compound 3 with those of 1 (Figures S4, S5, S20, and S21) showed that they differed from each other only in the signals of their side chains (Table 1 ). The proton and carbon signals of aglycon side chain of 3 demonstrated the presence of three methyl groups $\left(\delta_{\mathrm{H}} 1.53 \mathrm{~s}, 1.59 \mathrm{~s}, 1.65 \mathrm{~s} ; \delta_{\mathrm{C}} 28.5\right.$, $17.9,25.7)$ and one double bond $\left(\delta_{\mathrm{H}} 5.12 \mathrm{~m} ; \delta_{\mathrm{C}} 124.4,132.1\right)$. The protons sequence from $\mathrm{H}-22$ to H-27, correlated with the corresponding carbon atoms of the side chain of 3, was assigned using the COSY and HSQC experiments (Table 1, Figures S22 and S23). The key HMBC correlations $\mathrm{H}_{3}-21 / \mathrm{C}-17$, C-20, C-22; H-22/C-17, C-20; H-23/C-20, C-21; H-24/C-26, $\mathrm{C}-27 ; \mathrm{H}_{3}-26 / \mathrm{C}-24, \mathrm{C}-25, \mathrm{C}-27$, and $\mathrm{H}_{3}-27 / \mathrm{C}-24, \mathrm{C}-25, \mathrm{C}-26$ and key ROESY correlations $\mathrm{H}_{3}-$ 21/H-17, $\mathrm{H}-22$ and $\mathrm{H}-24 / \mathrm{H}_{3}-27, \mathrm{H}_{3}-26$ supported the total structure of the $\Delta^{24}$-lanostane side chain (Figure 1). Thus, aglycon of glycoside 3 was identical to aglycon of known cucumarioside $A_{1}$ from E. fraudatrix [25]. Therefore, the structure of pacificusoside $C$ (3) was established as $3 \beta-O-\{3-O-m e t h y l-\beta$-D-xylopyranosyl-( $(1 \rightarrow 3)-\beta$-D-glucopyranosyl- $(1 \rightarrow 4)-[\beta$-Dxylopyranosyl-( $(1 \rightarrow 2)$ ]- $\beta$-D-quinovopyranosyl-( $1 \rightarrow 2)-\beta$-D-xylopyranosyl\}-16 $\beta$-acetoxyholosta7,24-diene.

\subsection{In Vitro Anticancer Activity of Compounds 1-6}

The cytotoxicity of investigated compounds 1-6 was tested against human embryonic kidney HEK 293 cells, colorectal carcinoma HT-29 cells, melanoma RPMI-7951 cells, and breast cancer MDA-MB-231 cells using MTS assay. The results of the experiment are expressed in $\mathrm{IC}_{50}$ values (Table 3 ). The compounds $\mathbf{4} \mathbf{- 6}$ reveal high cytotoxic activity against the tested cell lines (Table 3 , column a). Compound 3 has comparable high cytotoxic activity against HEK $293\left(\mathrm{IC}_{50}=6.7 \pm 0.2\right)$, HT-29 $\left(\mathrm{IC}_{50}=6.2 \pm 0.3\right)$, and MDA-MB$231\left(\mathrm{IC}_{50}=6.4 \pm 0.4\right)$ cells and moderate effect against RPMI-7951 cells $\left(\mathrm{IC}_{50}=23.7 \pm 0.4\right)$. Compound 2 inhibits cell viability of all tested cell lines with $\mathrm{IC}_{50}$ value more than $20 \mu \mathrm{M}$ (Table 3, column a). In contrast, compound $\mathbf{1}$ was nontoxic against all types of cells at concentrations range up to $40 \mu \mathrm{M}$ (Table 3, column a). Cisplatin, a well-known chemotherapeutic drug, was used as the reference cytotoxic drug (positive control). $\mathrm{IC}_{50}$ of cisplatin against HEK 293, HT-29, RPMI-7951, and MDA-MB-231 cells was $64.6 \mu \mathrm{M}, 95.4 \mu \mathrm{M}$, $48.4 \mu \mathrm{M}$, and $7.8 \mu \mathrm{M}$, respectively, after $48 \mathrm{~h}$ of treatment (data not shown). 
Table 3. Cytotoxic activities of compounds 1-6 and their mixtures with cholesterol. Values are indicated as mean \pm standard deviation.

\begin{tabular}{|c|c|c|c|c|c|c|c|c|}
\hline \multirow{3}{*}{ Compound } & \multicolumn{8}{|c|}{ Inhibiting Concentration, $\left(\mathrm{IC}_{50}\right), \mu \mathrm{M}$} \\
\hline & \multicolumn{2}{|c|}{ HEK 293} & \multicolumn{2}{|c|}{ HT-29 } & \multicolumn{2}{|c|}{ RPMI-7951 } & \multicolumn{2}{|c|}{ MDA-MB-231 } \\
\hline & a & $\mathrm{b}$ & a & $\mathrm{b}$ & a & $\mathrm{b}$ & a & $\mathrm{b}$ \\
\hline 1 & $>40$ & n.d. & $>40$ & n.d. & $>40$ & n.d. & $>40$ & n.d. \\
\hline 2 & $28.6 \pm 2.1$ & $>40$ & $26.2 \pm 2.5$ & $>40$ & $20.4 \pm 1.6$ & $>40$ & $21.5 \pm 0.5$ & $>40$ \\
\hline 3 & $6.7 \pm 0.2$ & $24.2 \pm 4.0$ & $6.2 \pm 0.3$ & $19.4 \pm 3.3$ & $23.7 \pm 0.4$ & $>40$ & $6.4 \pm 0.4$ & $20.0 \pm 4.7$ \\
\hline 4 & $5.5 \pm 0.3$ & $17.3 \pm 0.8$ & $5.3 \pm 0.2$ & $14.3 \pm 3.0$ & $5.8 \pm 1.2$ & $16.4 \pm 3.1$ & $3.6 \pm 0.2$ & $12.8 \pm 0.7$ \\
\hline 5 & $7.9 \pm 0.9$ & $19.5 \pm 1.2$ & $5.5 \pm 0.6$ & $15.9 \pm 0.26$ & $5.9 \pm 0.9$ & $17.0 \pm 2.8$ & $4.4 \pm 0.09$ & $12.9 \pm 2.5$ \\
\hline 6 & $6.3 \pm 0.6$ & $13.2 \pm 0.5$ & $7.8 \pm 0.7$ & $10.9 \pm 0.05$ & $5.8 \pm 2.6$ & $11.4 \pm 0.8$ & $5.5 \pm 0.1$ & $10.5 \pm 1.8$ \\
\hline
\end{tabular}

$\mathrm{IC}_{50}$, the concentration of compounds that caused a $50 \%$ reduction in cell viability of tested normal and cancer cells. a IC 50 of individual compounds 1-6 for $24 \mathrm{~h}$. b IC 50 of 1-6 combined with cholesterol for $24 \mathrm{~h}$. n.d. - not determined.

Such potent cytotoxicity of compounds 3-6 can be explained by the fact that the studied triterpene glycosides are capable of interacting with cholesterol of cellular membranes, which leads to cell death as a result of disruption or loss of the integrity of the cell membrane. Since cholesterol is known for its function of stabilizing the cell membrane, the loss of cholesterol itself or the loss of its ability to perform this function is undoubtedly a reason of the observed instability of the cellular membranes and membranolysis of the target cells [26-28]. Since the triterpene saponins are able to complex cholesterol [29], we analyzed the influence of triterpene glycosides 2-6 combined with cholesterol on the cell viability. It was found that the cytotoxic activity of 2-6 combined with cholesterol was greatly reduced compared with activity of the investigated individual triterpene glycosides (Table 3, column b). The $\mathrm{IC}_{50}$ value of compounds $\mathbf{3} \mathbf{- 5}$ was increased by approximately 3 times, and compound 6-by 1.5 times (Table 3, column b). Obtained data suggested that mechanism of cytotoxic effect of triterpene glycosides 2-6 may be related to binding of membrane cholesterol resulting in membranolysis and cell death. Several works confirmed that triterpene glycosides from sea cucumbers possess potent membranolytic activity $[30,31]$.

It was demonstrated that the investigated compounds 1-6 (at concentrations 5, 10,20 , and $40 \mu \mathrm{M}$ ) did not show selective cytotoxicity on cancer cells, because the viability of normal cells was also suppressed. That is why we investigated the effect of compounds 1-6 on colony formation at the nontoxic concentrations of $0.1,0.5$, and $1 \mu \mathrm{M}$, at which they did not influence the viability of normal HEK 293 cells (data not shown).

Compounds 1, 2, and 6 at a concentration of $1 \mu \mathrm{M}$ possess moderate inhibitory activity on the model of the colony formation of the cancer cells, namely, 1, 2, and 6 decreased number of colonies of HT-29 by 21, 22, and 28\%, respectively; RPMI-7951by $27 \%, 26 \%$, and $45 \%$, respectively; MDA-MB-231-by 28,25 , and $40 \%$, respectively (Figure 3). Compounds 3, 4, and 5 almost completely suppressed colony growth of HT-29, RPMI-7951, and MDA-MB-231 cells at concentrations of $0.5 \mu \mathrm{M}$ and $1 \mu \mathrm{M}$ (Figure 3). To the best of our knowledge this is the first evidence that triterpene glycosides at nontoxic concentrations significantly inhibited the colony formation of human cancer cells. 
Н Т -29
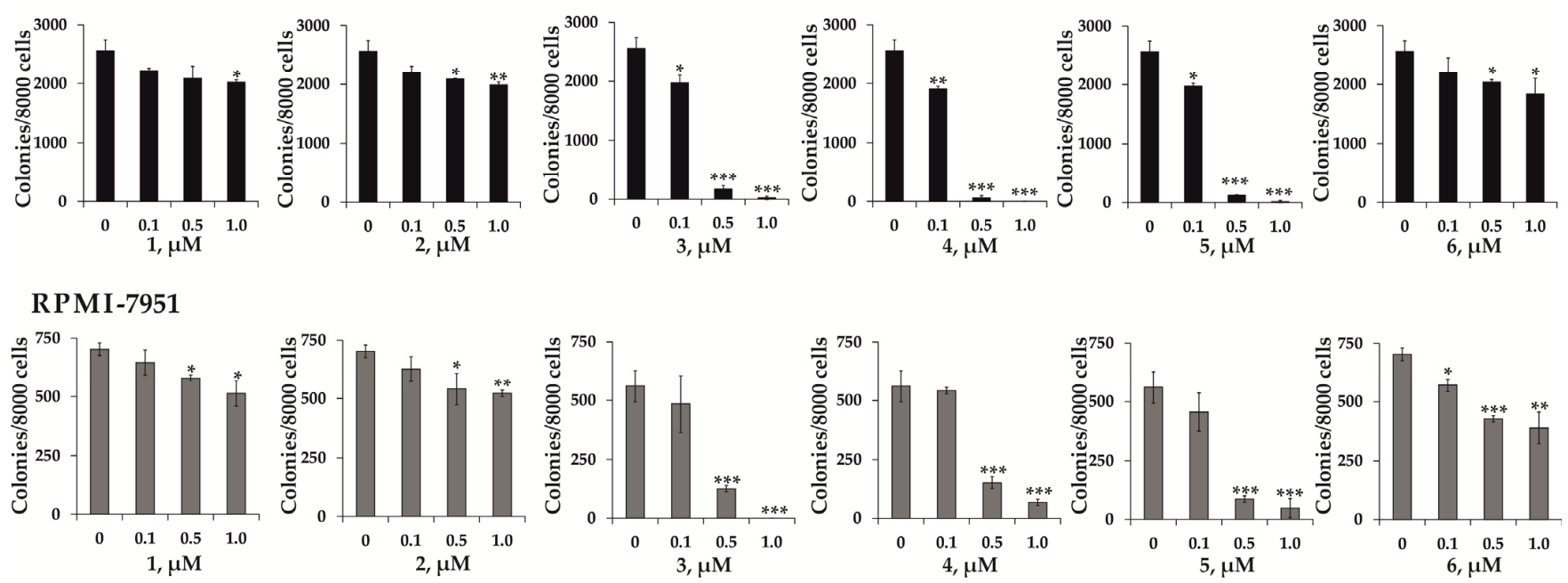

MDA-MB-231
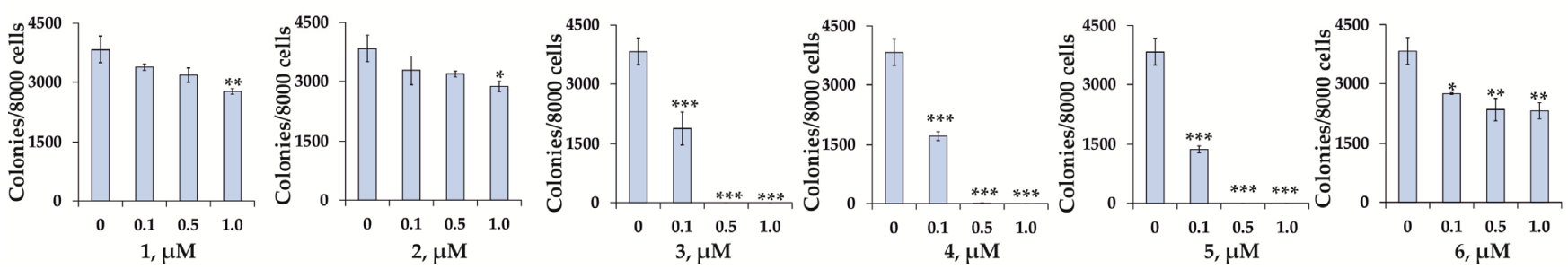

Figure 3. Effect of compounds 1-6 on colony formation of human colorectal carcinoma HT-29, melanoma RPMI-7951, and breast cancer MDA-MB-231 cell lines. HT-29, RPMI-7951, and MDA-MB-231 cells $\left(2.4 \times 10^{4} / \mathrm{mL}\right)$ treated with/without investigated 1-6 $(0.1,0.5$, and $1 \mu \mathrm{M})$ and subjected into a soft agar. The culture was maintained at $37{ }^{\circ} \mathrm{C}$ in a $5 \% \mathrm{CO}_{2}$ atmosphere for 2 weeks. The colonies were counted under a microscope with the aid of the ImageJ software program ( $n=6$ for control and each compound, $\mathrm{n}$-quantity of photos). The asterisks $\left.{ }^{*} p<0.05,{ }^{* *} p<0.01,{ }^{* * *} p<0.001\right)$ indicate a significant decrease in colony formation in cells treated with compounds compared with the nontreated cells (control).

\section{Conclusions}

The isolation of a series of new triterpene glycosides from starfish is a rare case. Previously, triterpene glycosides, characteristic metabolites of sea cucumbers, were isolated only from two species of starfish: A. rollestoni Bell and C. granulatus [20,21]. Pacificusosides A-C (1-3), new glycosides from the starfish S. pacificus have a great structural similarity with the triterpene glycosides earlier obtained from the sea cucumber E. fraudatrix. In particular, the isolated glycosides 1, 3 contain the holostane type of aglycons with $16 \beta$ acetoxy group and 7(8)-double bond and glycoside 2 contains the rare nonholostane type aglycon, 23,24,25,26,27-pentanorlanosta-7,20(22)-diene-18(16)-lactone-3 $\beta$-ol.

The oligoglycoside chains of 1-3 have 3-O-methyl-D-xylose as a terminal monosaccharide unit. This monosaccharide residue was previously found only in the sea cucumber $E$. fraudatrix. It was previously suggested that this structural peculiarity is a chemotaxonomic marker of E. fraudatrix [32]. The finding of 3-O-methyl-D-xylose unit in pacificusosides A-C (1-3) from the starfish shows that these glycosides are food markers. Probably, these compounds are partly metabolized in the starfish glycosides from the sea cucumber, belonging to the genus Eupentacta and obtained with diet. The studied starfish has the synonymized name: Solaster endeca pacificus (Djakonov, 1938). It was reported that in the Atlantic Ocean S. endeca pacificus feeds on other starfish and bivalve mollusks, but in the Pacific, its diet is mainly sea cucumbers and other invertebrates [33]. Without doubt, the starfish S. pacificus feeds on sea cucumbers, as was reported earlier.

At the same time, glycoside 1 has a novel unique type of side chain, containing the aldehyde group in the triterpene aglycon. This structural fragment was not previously 
found in the sea cucumber triterpene glycosides or sea star steroidal glycosides. Obviously, pacificusoside $\mathrm{A}$ is a metabolite, formed by the transformation of a toxic glycoside from a diet in the starfish. It is of particular interest that modified glycoside (1) is significantly less cytotoxic when compared with intact cucumariosides from E. fraudatrix. We assumed that the formation of an unusual glycoside, pacificusoside A (1), from related glycosides of the starfish is carried out in two stages. At the first stage, cucumarioside $\mathrm{C}_{2}$ (5) from the sea cucumber E. fraudatrix, most probably the biosynthetic precursor of pacificusoside A (1), is oxidized by oxygenases similar to $P_{450}$ cytochromes. At the second stage, the shortening of the $\Delta^{22,24}$-lanostane side chain of compound 5 occurs in the starfish S. pacificus with the formation of the $\Delta^{22}$-24-aldo-25,26,27-trinorlanostane side chain in glycoside 1 (Figure 4). Presumably, the cleavage of part of the side chain occurs under the action of specific oxygenases, similarly to the formation of pregnenolone from cholesterol under the action of cytochrome $\mathrm{P} 450_{\mathrm{scc}}$ in mammals during the synthesis of progesterone. The existence of such oxygenases in sea stars can be confirmed by the detection of (24R)-5 $\alpha$ cholestane- $3 \beta, 4 \beta, 6 \beta, 8,15 \beta, 24,25$-heptaol, which has hydroxy groups at C-24 and C-25 in cholestane side chain [34] as in the intermediate on Figure 4.

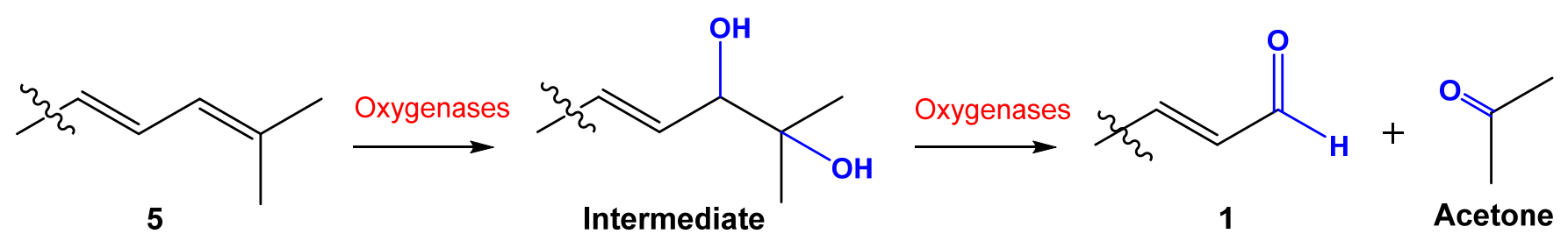

Figure 4. Hypothetic scheme of the biosynthesis of aglycon side chain of compound $\mathbf{1}$.

We believe that the formation of similar compounds with an aldehyde group in the side chain of glycosides 3 and 4 is also possible, but requires more time after feeding. Moreover, the slow metabolism of the toxic triterpene glycosides in starfish allows them to use the accumulated dietary ichthyotoxic compounds to defend themselves against predators.

Thus, the oxidation with a subsequent decrease in the number of carbon atoms in the side chain of the triterpene glycosides partly led to the decrease in the cytotoxicity of the modified compounds. Indeed, based on the obtained data, one can see a pattern between the structure and biological activity of the isolated compounds. The highest cytotoxicity against all types of cancer and normal cells, as well as the highest inhibitory effect on colony formation of cancer cells, were demonstrated by glycosides 3-5, which have $\Delta^{24}$-lanostane and cis- and trans- $\Delta^{22,24}$-lanostane side chains in the triterpene aglycon, respectively. At the same time, the $\mathrm{IC}_{50}$ of pacificusoside A (1) on all cell types was more than $40 \mu \mathrm{M}$, and this compound also slightly inhibited the colony formation of all types of cancer cells in a soft agar assay. According to the data of the colony formation assay, the glycosides 2 and 6 , which have a triterpene aglycon with a rearranged 18(16)-lactone, are less active than glycosides 3-5, which have a holostane triterpene aglycon with an 18(20)-lactone.

Finally, we once more confirmed the hypothesis that triterpene glycosides are membranolytic and exert their cytotoxic effect by binding the components of the plasma membrane of target cells. In fact, when cholesterol was combined with the compounds 1-6, the cytotoxicity of all glycosides decreased markedly. This is likely due to the fact that some of the molecules of triterpene glycosides 1-6 bind the cholesterol added to these compounds.

On the other hand, glycosides $3-5$, which showed significant or complete inhibition the colony formation of cancer cells at nontoxic concentrations, can be further studied as potential anticancer agents.

Supplementary Materials: The following are available online at https:/ / www.mdpi.com/2218-273 X/11/3/427/s1, Figure S1: (-)HRESIMS spectrum of pacificusoside A (1); Figure S2: IR spectrum of pacificusoside A (1) in KBr; Figure S3: UV spectrum of pacificusoside A (1) in MeOH; Figure S4: ${ }^{1} \mathrm{H}$ NMR spectrum of pacificusoside $A(1)$ in $\mathrm{C}_{5} \mathrm{D}_{5} \mathrm{~N}$; Figure $\mathrm{S} 5:{ }^{13} \mathrm{C}$ NMR spectrum of pacificusoside $\mathrm{A}$ 
(1) in $\mathrm{C}_{5} \mathrm{D}_{5} \mathrm{~N}$; Figure $\mathrm{S6}:{ }^{1} \mathrm{H}_{-}{ }^{1} \mathrm{H}$ COSY spectrum of pacificusoside A (1) in $\mathrm{C}_{5} \mathrm{D}_{5} \mathrm{~N}$; Figure S7: HSQC spectrum of pacificusoside A (1) in $\mathrm{C}_{5} \mathrm{D}_{5} \mathrm{~N}$; Figure S8: HMBC spectrum of pacificusoside A (1) in $\mathrm{C}_{5} \mathrm{D}_{5} \mathrm{~N}$; Figure S9: ROESY spectrum of pacificusoside A (1) in $\mathrm{C}_{5} \mathrm{D}_{5} \mathrm{~N}$; Figure S10: (-)HRESIMS spectrum of pacificusoside B (2); Figure S11: IR spectrum of pacificusoside B (2) in KBr; Figure S12: ${ }^{1} \mathrm{H}$ NMR spectrum of pacificusoside $\mathrm{B}(2)$ in $\mathrm{C}_{5} \mathrm{D}_{5} \mathrm{~N}$; Figure $\mathrm{S13}:{ }^{13} \mathrm{C}$ NMR spectrum of pacificusoside $\mathrm{B}$ (2) in $\mathrm{C}_{5} \mathrm{D}_{5} \mathrm{~N}$; Figure S14: ${ }^{1} \mathrm{H}-{ }^{1} \mathrm{H}-\mathrm{COSY}$ spectrum of pacificusoside $\mathrm{B}(2)$ in $\mathrm{C}_{5} \mathrm{D}_{5} \mathrm{~N}$; Figure S15: HSQC spectrum of pacificusoside B (2) in $\mathrm{C}_{5} \mathrm{D}_{5} \mathrm{~N}$; Figure S16: HMBC spectrum of pacificusoside B (2) in $\mathrm{C}_{5} \mathrm{D}_{5} \mathrm{~N}$; Figure S17: ROESY spectrum of pacificusoside B (2) in $\mathrm{C}_{5} \mathrm{D}_{5} \mathrm{~N}$; Figure S18: (-)HRESIMS spectrum of pacificusoside C (3); Figure S19: IR spectrum of pacificusoside C (3) in KBr, Figure S20: ${ }^{1} \mathrm{H}$ NMR spectrum of pacificusoside $\mathrm{C}(3)$ in $\mathrm{C}_{5} \mathrm{D}_{5} \mathrm{~N}$; Figure $\mathrm{S} 21$ : ${ }^{13} \mathrm{C}$ NMR spectrum of pacificusoside $\mathrm{C}$ (3) in $\mathrm{C}_{5} \mathrm{D}_{5} \mathrm{~N}$; Figure S22: ${ }^{1} \mathrm{H}-{ }^{1} \mathrm{H}$ COSY spectrum of pacificusoside $\mathrm{C}$ (3) in $\mathrm{C}_{5} \mathrm{D}_{5} \mathrm{~N}$; Figure S23: HSQC spectrum of pacificusoside $C_{\text {; }}(3)$ in $\mathrm{C}_{5} \mathrm{D}_{5} \mathrm{~N}$; Figure S24: HMBC spectrum of pacificusoside $C_{\text {; }}$ (3) in $\mathrm{C}_{5} \mathrm{D}_{5} \mathrm{~N}$; Figure S25: ROESY spectrum of pacificusoside $\mathrm{C}$; (3) in $\mathrm{C}_{5} \mathrm{D}_{5} \mathrm{~N}$.

Author Contributions: T.V.M. isolated the metabolites, elucidated their structure, and prepared the manuscript; A.A.K. and N.V.I. analyzed the compounds and edited the manuscript; A.I.K. performed the acquisition and interpretation of NMR spectra; P.S.D. did the acquisition and interpretation of mass spectra; A.S.K. investigated the cytotoxic and anticancer activity of the compounds; O.S.M. described the results of the biological activity of the compounds and prepared the manuscript.; V.A.S. edited the manuscript. All authors have read and agreed to the published version of the manuscript.

Funding: The isolation and establishment of the chemical structures were partially supported by Grant No. 20-03-00014 from the RFBR (Russian Foundation for Basic Research). The study on the cytotoxic and the anticancer activities of starfish metabolites was supported by Grant No. 18-74-10028 from the RSF (Russian Science Foundation).

Institutional Review Board Statement: Not applicable.

Informed Consent Statement: Not applicable.

Data Availability Statement: The data presented in this study are available on request from the corresponding authors.

Acknowledgments: The study was carried out on the equipment of the Collective Facilities Center "The Far Eastern Center for Structural Molecular Research (NMR/MS) of PIBOC FEB RAS". We are grateful to B.B. Grebnev (G.B. Elyakov Pacific Institute of Bioorganic Chemistry FEB RAS, Vladivostok, Russia) for species identification of the starfish, V.I. Kalinin for reading and discussion of the manuscript, E.V. Evtushenko for providing sample of 3-O-Me-Xyl, V.P. Glazunov for providing IR spectra of compounds and N.Y. Kim for providing UV spectra of compounds.

Conflicts of Interest: The authors declare no conflict of interest.

\section{References}

1. Gomes, A.R.; Freitas, A.C.; Rocha-Santos, T.A.P.; Duarte, A.C. Bioactive compounds derived from echinoderms. RSC Adv. 2014, 4, 29365-29382. [CrossRef]

2. Mondol, M.A.M.; Shin, H.J.; Rahman, M.A.; Islam, M.T. Sea cucumber glycosides: Chemical structures, producing species and important biological properties. Mar. Drugs 2017, 15, 317. [CrossRef] [PubMed]

3. Kalinin, V.I.; Silchenko, A.S.; Avilov, S.A.; Stonik, V.A. Non-holostane aglycones of sea cucumber triterpene glycosides. Structure, biosynthesis, evolution. Steroids 2019, 147, 42-51. [CrossRef]

4. Kalinin, V.I.; Aminin, D.L.; Avilov, S.A.; Silchenko, A.S.; Stonik, V.A. Triterpene glycosides from sea cucumbers (Holothurioidea, Echinodermata), biological activities and functions. In Studies in Natural Product Chemistry (Bioactive Natural Products); Atta-urRahman, Ed.; Elsevier Science Publisher: Amsterdam, The Netherlands, 2008; Volume 35, pp. 135-196.

5. Careaga, V.P.; Maier, M.S. Cytotoxic triterpene glycosides from sea cucumbers. In Handbook of Anticancer Drugs from Marine Origin; Kim, S.-K., Ed.; Springer International Publishing: Cham, Switzerland, 2015; pp. 515-528.

6. Chludil, H.D.; Murray, A.P.; Seldes, A.M.; Maier, M.S. Biologically active triterpene glycosides from sea cucumbers (Holothuroidea, Echinodermata). In Studies in Natural Products Chemistry; Atta-ur-Rahman, Ed.; Elsevier Science Publisher: Amsterdam, The Netherlands, 2003; Volume 28, pp. 587-615.

7. Kim, S.K.; Himaya, S.W.A. Chapter 20-Triterpene glycosides from sea cucumbers and their biological activities. Adv. Food Nutr. Res. 2012, 63, 297-319. [CrossRef]

8. Maier, M.S.; Roccatagliata, A.J.; Kuriss, A.; Chludil, H.; Seldes, A.M.; Pujol, C.A.; Damonte, E.B. Two new cytotoxic and virucidal trisulfated triterpene glycosides from the Antarctic sea cucumber Staurocucumis liouvillei. J. Nat. Prod. 2001, 64, 732-736. [CrossRef] 
9. Aminin, D.L.; Menchinskaya, E.S.; Pisliagin, E.A.; Silchenko, A.S.; Avilov, S.A.; Kalinin, V.I. Anticancer activity of sea cucumber triterpene glycosides. Mar. Drugs 2015, 13, 1202-1223. [CrossRef]

10. Aminin, D.L.; Pislyagin, E.A.; Menchinskaya, E.S.; Silchenko, A.S.; Avilov, S.A.; Kalinin, V.I. Immunomodulatory and anticancer activity of sea cucumber triterpene glycosides. In Studies in Natural Products Chemistry (Bioactive Natural Products); Atta-ur-Rahman, Ed.; Elsevier Science Publisher: Amsterdam, The Netherlands, 2014; Volume 41, pp. 75-94.

11. Minale, L.; Riccio, R.; Zollo, F. Steroidal oligoglycosides and polyhydroxysteroids from Echinoderms. Fortschr. Chem. Org. Nat. 1993, 62, 75-308. [CrossRef]

12. Stonik, V.A. Marine polar steroids. Russ. Chem. Rev. 2001, 70, 673-715. [CrossRef]

13. Iorizzi, M.; De Marino, S.; Zollo, F. Steroidal oligoglycosides from the Asteroidea. Curr. Org. Chem. 2001, 5, 951-973. [CrossRef]

14. Stonik, V.A.; Ivanchina, N.V.; Kicha, A.A. New polar steroids from starfish. Nat. Prod. Commun. 2008, 3, 1587-1610. [CrossRef]

15. Dong, G.; Xu, T.H.; Yang, B.; Lin, X.P.; Zhou, X.F.; Yang, X.W.; Liu, Y.H. Chemical constituents and bioactivities of starfish. Chem. Biodivers. 2011, 8, 740-791. [CrossRef]

16. Ivanchina, N.V.; Kicha, A.A.; Stonik, V.A. Steroid glycosides from marine organisms. Steroids 2011, 76, 425-454. [CrossRef] [PubMed]

17. Ivanchina, N.V.; Kicha, A.A.; Malyarenko, T.V.; Stonik, V.A. Advances in Natural Products Discovery; Gomes, A.R., Rocha-Santos, T., Duarte, A., Eds.; Nova Science Publishers: New York, NY, USA, 2017; Volume 6, pp. 191-224.

18. Stonik, V.A.; Kicha, A.A.; Malyarenko, T.V.; Ivanchina, N.V. Asterosaponins: Structures, taxonomic distribution, biogenesis and biological activities. Mar. Drugs 2020, 18, 584. [CrossRef]

19. Xia, J.M.; Miao, Z.; Xie, C.L.; Zhang, J.W.; Yang, X.W. Chemical constituents and bioactivities of starfish: An update. Chem. Biodivers. 2020, 17, e1900638. [CrossRef]

20. Zhan, Y.C.; Sun, Y.; Li, W.; Lin, Y.; Sha, Y.; Pei, Y.H. A new triterpene glycoside from Asterias rollentoni. J. Asian Nat. Prod. Res. 2006, 8, 631-636. [CrossRef]

21. Ivanchina, N.V.; Kalinovsky, A.I.; Malyarenko, T.V.; Kicha, A.A.; Dmitrenok, P.S. A holothurian triterpene glycoside holothurin $\mathrm{A}_{2}$ (= echinoside A) isolated from the starfish Choriaster granulatus. Nat. Prod. Commun. 2019, 14, 1-3. [CrossRef]

22. Afiyatullov, S.S.; Kalinovsky, A.I.; Stonik, V.A. Structures of cucumariosides $\mathrm{C}_{1}$ and $\mathrm{C}_{2}$-two new triterpene glycosides from the holothurian Eupentacta fraudatrix. Chem. Nat. Comp. 1987, 23, 691-696. [CrossRef]

23. Silchenko, A.S.; Kalinovsky, A.I.; Avilov, S.A.; Andrijaschenko, P.V.; Dmitrenok, P.S.; Martyyas, E.A.; Kalinin, V.I. Triterpene glycosides from the sea cucumber Eupentacta fraudatrix. Structure and cytotoxic action of cucumariosides $A_{2}, A_{7}, A_{9}, A_{10}, A_{11}$, $\mathrm{A}_{13}$ and $\mathrm{A}_{14}$, seven new minor non-sulfated tetraosides and an aglycone with an uncommon 18-hydroxy group. Nat. Prod. Commun. 2012, 7, 845-852. [CrossRef]

24. Silchenko, A.S.; Kalinovsky, A.I.; Avilov, S.A.; Andryjaschenko, P.V.; Dmitrenok, P.S.; Martyyas, E.A.; Kalinin, V.I. Triterpene glycosides from the sea cucumber Eupentacta fraudatrix. Structure and biological action of cucumariosides $\mathrm{I}_{1}, \mathrm{I}_{3}, \mathrm{I}_{4}$, three new minor disulfated pentaosides. Nat. Prod. Commun. 2013, 8, 1053-1058. [CrossRef] [PubMed]

25. Silchenko, A.S.; Kalinovsky, A.I.; Avilov, S.A.; Andryjaschenko, P.V.; Dmitrenok, P.S.; Martyyas, E.A.; Kalinin, V.I. Triterpene glycosides from the sea cucumber Eupentacta fraudatrix. Structure and biological action of cucumariosides $\mathrm{A}_{1}, \mathrm{~A}_{3}, \mathrm{~A}_{4}, \mathrm{~A}_{5}, \mathrm{~A}_{6}, \mathrm{~A}_{12}$ and $\mathrm{A}_{15}$, seven new minor non-sulfated tetraosides and unprecedented 25-keto, 27-norholostane aglycone. Nat. Prod. Commun. 2012, 7, 517-525. [CrossRef]

26. Schlösser, E. Interaction of saponins with cholesterol, lecithin, and albumin. Can. J. Physiol. Pharmacol. 1969, 47, 487-490. [CrossRef] [PubMed]

27. De Geyter, E.; Swevers, L.; Soin, T.; Geelen, D.; Smagghe, G. Saponins do not affect the ecdysteroid receptor complex but cause membrane permeation in insect culture cell lines. J. Insect. Physiol. 2012, 58, 18-23. [CrossRef]

28. Böttger, S.; Melzig, M.F. The influence of saponins on cell membrane cholesterol. Bioorg. Med. Chem. 2013, 21, 7118-7124. [CrossRef] [PubMed]

29. Wojciechowski, K.; Orczyk, M.; Gutberlet, T.; Geue, T. Complexation of phospholipids and cholesterol by triterpenic saponins in bulk and in monolayers. Biochim. Biophys. Acta Biomembr. 2016, 1858, 363-373. [CrossRef]

30. Wang, J.; Han, H.; Chen, X.; Yi, Y.; Sun, H. Cytotoxic and apoptosis-inducing activity of triterpene glycosides from Holothuria scabra and Cucumaria frondosa against HepG2 cells. Mar. Drugs 2014, 12, 4274-4290. [CrossRef] [PubMed]

31. Popov, A.M.; Kalinovskaia, N.I.; Kuznetsova, T.A.; Agafonova, I.G.; Anisimov, M.M. Role of sterols in the membranotropic activity of triterpene glycosides. Antibiotiki 1983, 28, 656-659.

32. Popov, R.S.; Ivanchina, N.V.; Silchenko, A.S.; Avilov, S.A.; Kalinin, V.I.; Dolmatov, I.Y.; Stonik, V.A.; Dmitrenok, P.S. Metabolite profiling of triterpene glycosides of the Far Eastern sea cucumber Eupentacta fraudatrix and their distribution in various body components using LC-ESI QTOF-MS. Mar. Drugs 2017, 15, 302. [CrossRef]

33. Sea Stars of the Pacific Northwest. Available online: https://seastarsofthepacificnorthwest.info/northern-sun-star.html (accessed on 16 February 2021).

34. Ivanchina, N.V.; Kicha, A.A.; Kalinovsky, A.I.; Dmitrenok, P.S.; Dmitrenok, A.S.; Chaikina, E.L.; Stonik, V.A.; Gavagin, M.; Cimino, G. Polar steroids compounds from the Far Eastern starfish Henricia leviuscula. J. Nat. Prod. 2006, 69, 224-228. [CrossRef] 